\title{
Regio- and stereoselective synthesis of alkenylphosphines: a rhodium-catalyzed hydrophosphination of alkynes using a silylphosphine
}

\author{
Minoru Hayashi,* Yutaka Matsuura, and Yutaka Watanabe \\ Department of Materials Science and Biotechnology, \\ Graduate School of Science and Engineering, Ehime University \\ 3 Bunkyo-cho, Matsuyama 790-8577, Japan \\ E-mail: hayashi@eng.ehime-u.ac.jp
}

Supporting Information

\section{Contents}

$\begin{array}{ll}\text { General information } & \text { S2 }\end{array}$

$\begin{array}{ll}\text { General procedure } & \text { S2 }\end{array}$

$\begin{array}{ll}\text { Characterization data for } \mathbf{3 a}-\mathbf{i}, \mathbf{5}, \mathbf{7} & \text { S3-S7 }\end{array}$

$\begin{array}{ll}\text { References } & \text { S8 }\end{array}$

${ }^{1} \mathrm{H},{ }^{13} \mathrm{C}$ and ${ }^{31} \mathrm{P}$ NMR Spectra of Compounds $\mathbf{3 a - i ,}, \mathbf{5}, \mathbf{7} \quad$ S9-S24 


\section{General information}

Unless otherwise noted, $\mathrm{CDCl}_{3}$ was used as solvent for ${ }^{1} \mathrm{H},{ }^{13} \mathrm{C},{ }^{31} \mathrm{P}$ NMR spectra $(400 \mathrm{MHz}$ for ${ }^{1} \mathrm{H}, 100 \mathrm{MHz}$ for ${ }^{13} \mathrm{C}$ and $162 \mathrm{MHz}$ for ${ }^{31} \mathrm{P}$ ). HR-FABMS spectra were collected using PEG-200 as a matrix and internal reference.

Silylphosphines 1a and $\mathbf{1 b}$ were prepared by the reported procedure. ${ }^{1}$ Benzene was dried by distillation over $\mathrm{Na}$. $\mathrm{MeOH}$ was purified by distillation over $\mathrm{Mg}(\mathrm{OMe})_{2}$ generated in situ. $[\mathrm{Rh}(\operatorname{cod}) \mathrm{Cl}]_{2}$ was prepared by the reported procedure. ${ }^{2}$ AgOTf was purchased from commercial supplier and was dried just before use in vacuo over $\mathrm{P}_{2} \mathrm{O}_{5}\left(140^{\circ} \mathrm{C} / 0.1 \mathrm{mmHg}, 2 \mathrm{~h}\right)$. Other reagents were purchased from commercial suppliers and used after purification (distillation).

The following complexes, additives and ligands were tested in the catalyst survey: complexes $\left(\mathrm{Ru}_{3}(\mathrm{CO})_{12}, \mathrm{RuCl}_{2}\left(\mathrm{PPh}_{3}\right)_{3}, \mathrm{RhCl}\left(\mathrm{PPh}_{3}\right)_{3},[\mathrm{Rh}(\mathrm{cod}) \mathrm{Cl}]_{2},\left[\mathrm{Rh}(\mathrm{cod})_{2}\right] \mathrm{BF}{ }_{4}, \mathrm{Pd}\left(\mathrm{PPh}_{3}\right)_{4}, \mathrm{PdCl}_{2}\left(\mathrm{PPh}_{3}\right)_{2}\right.$, $\left.\mathrm{PdCl}_{2}\left(\mathrm{CH}_{3} \mathrm{CN}\right)_{2}\right)$, phosphine ligands $\left(\mathrm{PPh}_{3}, \mathrm{P}^{n} \mathrm{Bu}_{3}\right.$, dppe, dppf), silver salts $\left(\mathrm{AgOTf}, \mathrm{AgBF}_{4}\right)$, alcohols $\left(\mathrm{CH}_{3} \mathrm{OH}, \mathrm{PhCH}_{2} \mathrm{CH}_{2} \mathrm{CH}_{2} \mathrm{OH}\right)$.

\section{General procedure}

To a solution of $[\mathrm{Rh}(\operatorname{cod}) \mathrm{Cl}]_{2}(6 \mathrm{mg}, 12.5 \mu \mathrm{mol})$ and $\mathrm{MeOH}(20 \mu \mathrm{L})$ in anhydrous benzene (2 $\mathrm{mL}$ ) was added silver triflate $(6 \mathrm{mg}, 25 \mu \mathrm{mol})$ under argon. After the mixture was stirred for 20 min at r.t., alkyne $(0.50 \mathrm{mmol})$ and silylphosphine $(0.6 \mathrm{mmol})$ were added successively. The mixture turned red with the addition of silylphosphine. The resulting mixture was heated under reflux until the color of the mixture turned from dark red to yellow (about $8-10 \mathrm{~h}$ ). After the reaction mixture was concentrated, purification of the crude product by column chromatography 
on silica gel afforded the corresponding alkenylphosphine.

\section{Characterization Data}

The alkenylphosphines $\mathbf{3 a},{ }^{3} \mathbf{3 f},{ }^{4} \mathbf{3 g},{ }^{5} \mathbf{5},{ }^{4} \mathbf{7}^{4}$ were known compounds.

\section{(E)- $\beta$-Diphenylphosphinylstyrene (3a)}

This compound was synthesized following the general procedure from ethynylbenzene (51 $\mathrm{mg}$,

$0.5 \mathrm{mmol}$ ). Flash chromatography (eluent: AcOEt $/ \mathrm{n}-\mathrm{Hexane}=1 / 10, \mathrm{R}_{\mathrm{f}} 0.5$ ) yielded $128 \mathrm{mg}$ (89\%) as colorless oil. IR (neat) 2970, 2950, 1654, 1257, 1100, $1066 \mathrm{~cm}^{-1}$; ${ }^{1} \mathrm{H}$ NMR $\delta 6.85$ (dd, $J=12.8,22.8 \mathrm{~Hz}, 1 \mathrm{H}), 7.29-7.40(\mathrm{~m}, 10 \mathrm{H}), 7.47(\mathrm{dd}, J=12.8,22.8 \mathrm{~Hz}, 1 \mathrm{H}), 7.49-7.58(\mathrm{~m}, 5$ $\mathrm{H}) ;{ }^{13} \mathrm{C}$ NMR $\delta 119.3\left(\mathrm{~d}, J_{\mathrm{C}-\mathrm{P}}=96.3 \mathrm{~Hz}\right), 127.8,128.6\left(\mathrm{~d}, J_{\mathrm{C}-\mathrm{P}}=12.8 \mathrm{~Hz}\right), 129.0,130.1,131.4$, $131.9,133.2\left(\mathrm{~d}, J_{\mathrm{C}-\mathrm{P}}=96.5 \mathrm{~Hz}\right), 135.1\left(\mathrm{~d}, J_{\mathrm{C}-\mathrm{P}}=18.9 \mathrm{~Hz}\right), 147.5\left(\mathrm{~d}, J_{\mathrm{C}-\mathrm{P}}=3.7 \mathrm{~Hz}\right) ;{ }^{31} \mathrm{P}\left\{{ }^{1} \mathrm{H}\right\} \mathrm{NMR}$ $\delta$-10.9. HRMS calcd. for $\mathrm{C}_{20} \mathrm{H}_{18} \mathrm{OP}$ (phosphine oxide) $305.1095[\mathrm{M}+\mathrm{H}]^{+}$, found: 305.1135 .

\section{(E)- $\beta$-Diphenylphosphinyl-4-methoxystyrene (3b)}

This compound was synthesized following the general procedure from 4-ethynylanisole (66 mg, $0.5 \mathrm{mmol}$ ). Flash chromatography (eluent: $\left.\mathrm{CHCl}_{3} / \mathrm{n}-\mathrm{Hexane}=1 / 4, \mathrm{R}_{\mathrm{f}} 0.4\right)$ yielded $84 \mathrm{mg}(53 \%)$ as colorless solid, mp $141-144{ }^{\circ} \mathrm{C}$. IR (neat) $3020,2923,1554,1121,1002 \mathrm{~cm}^{-1} ;{ }^{1} \mathrm{H}$ NMR $\delta$ $3.80(\mathrm{~s}, 3 \mathrm{H}), 6.33(\mathrm{dd}, J=2.8,12.8 \mathrm{~Hz}, 1 \mathrm{H}), 6.85(\mathrm{~d}, J=12.8 \mathrm{~Hz}, 1 \mathrm{H}), 7.26-7.45(\mathrm{~m}, 14 \mathrm{H}) ;{ }^{13} \mathrm{C}$ NMR $\delta 55.3,113.6,126.8\left(\mathrm{~d}, J_{\mathrm{C}-\mathrm{P}}=15.8 \mathrm{~Hz}\right), 128.5\left(\mathrm{~d}, J_{\mathrm{C}-\mathrm{P}}=5.2 \mathrm{~Hz}\right), 128.6,\left(\mathrm{~d}, J_{\mathrm{C}-\mathrm{P}}=6.7 \mathrm{~Hz}\right)$, $129.8\left(\mathrm{~d}, J_{\mathrm{C}-\mathrm{P}}=2.2 \mathrm{~Hz}\right), 131.1\left(\mathrm{~d}, J_{\mathrm{C}-\mathrm{P}}=9.0 \mathrm{~Hz}\right), 132.7\left(\mathrm{~d}, J_{\mathrm{C}-\mathrm{P}}=18.8 \mathrm{~Hz}\right), 139.5\left(\mathrm{~d}, J_{\mathrm{C}-\mathrm{P}}=9.4\right.$ 
$\mathrm{Hz}), 143.6\left(\mathrm{~d}, J_{\mathrm{C}-\mathrm{P}}=19.2 \mathrm{~Hz}\right), 159.6\left(\mathrm{~d}, J_{\mathrm{C}-\mathrm{P}}=0.8 \mathrm{~Hz}\right) ;{ }^{31} \mathrm{P}\left\{{ }^{1} \mathrm{H}\right\} \mathrm{NMR} \delta-24.0$. Elemental analysis calcd. for $\mathrm{C}_{21} \mathrm{H}_{19} \mathrm{OP}: \mathrm{C}, 79.23 ; \mathrm{H}, 6.02$, found: $\mathrm{C}, 78.84 ; \mathrm{H}, 6.02$.

\section{(E)-1-Diphenylphosphinyl-1-heptene (3c)}

This compound was synthesized following the general procedure from 1-heptyne (48 mg, 0.5 mmol). Flash chromatography (eluent: $\left.\mathrm{CHCl}_{3} / \mathrm{n}-\mathrm{Hexane}=1 / 10, \mathrm{R}_{\mathrm{f}} 0.5\right)$ yielded $110 \mathrm{mg}(78 \%)$ as colorless oil. IR (neat) 613,1201, 1536, $2989 \mathrm{~cm}^{-1}$; ${ }^{1} \mathrm{H}$ NMR $\delta 0.85(\mathrm{t}, J=6.8 \mathrm{~Hz}, 3 \mathrm{H}), 1.31$ $(\mathrm{m}, 2 \mathrm{H}), 1.45(\mathrm{~m}, 2 \mathrm{H}), 2.21(\mathrm{q}, J=7.2 \mathrm{~Hz}, 2 \mathrm{H}), 6.11-6.32(\mathrm{~m}, 2 \mathrm{H}), 7.41-7.52(\mathrm{~m}, 6 \mathrm{H})$, 7.73-7.78 (m, 4H); ${ }^{13} \mathrm{C}$ NMR $\delta 13.9\left(\mathrm{~d}, J_{\mathrm{C}-\mathrm{P}}=9.4 \mathrm{~Hz}\right), 22.3\left(\mathrm{~d}, J_{\mathrm{C}-\mathrm{P}}=13,7 \mathrm{~Hz}\right), 28.0\left(\mathrm{~d}, J_{\mathrm{C}-\mathrm{P}}=\right.$ $11.4 \mathrm{~Hz}), 28.9\left(\mathrm{~d}, J_{\mathrm{C}-\mathrm{P}}=15,6 \mathrm{~Hz}\right), 29.2\left(\mathrm{~d}, J_{\mathrm{C}-\mathrm{P}}=25.8 \mathrm{~Hz}\right), 31.2\left(\mathrm{~d}, J_{\mathrm{C}-\mathrm{P}}=28,5 \mathrm{~Hz}\right), 128.5\left(\mathrm{~d}, J_{\mathrm{C}-\mathrm{P}}\right.$ $=11.9 \mathrm{~Hz}), 131.4\left(\mathrm{~d}, J_{\mathrm{C}-\mathrm{P}}=10.4 \mathrm{~Hz}\right), 131.4\left(\mathrm{~d}, J_{\mathrm{C}-\mathrm{P}}=2.9 \mathrm{~Hz}\right), 132.8\left(\mathrm{~d}, J_{\mathrm{C}-\mathrm{P}}=80.6 \mathrm{~Hz}\right), 133.4(\mathrm{~d}$, $\left.J_{\mathrm{C}-\mathrm{P}}=71.1 \mathrm{~Hz}\right), 147.1\left(\mathrm{~d}, J_{\mathrm{C}-\mathrm{P}}=12.1 \mathrm{~Hz}\right) ;{ }^{31} \mathrm{P}\left\{{ }^{1} \mathrm{H}\right\}$ NMR $\delta-12.8 . \quad$ HRMS calcd. for $\mathrm{C}_{19} \mathrm{H}_{24} \mathrm{P}$ : $283.1616[\mathrm{M}+\mathrm{H}]^{+}$, found: 283.1602 .

\section{(E)-1-Diphenylphosphinyl-3-hydroxy-1-propene (3d)}

This compound was synthesized following the general procedure from 2-propyn-1-ol (28 mg, 0.5 mmol). Flash chromatography (eluent: AcOEt $/ \mathrm{n}-$ Hexane $=1 / 4, \mathrm{R}_{\mathrm{f}} 0.3$ ) yielded $80 \mathrm{mg}(66 \%)$ as colorless oil. IR (neat) 791, 1298, 1554, 3004, $3420 \mathrm{~cm}^{-1} ;{ }^{1} \mathrm{H}$ NMR $\delta 2.25$ (br, $\left.1 \mathrm{H}\right), 4.48$ (d, $J=$ $6.0 \mathrm{~Hz}, 2 \mathrm{H}), 6.37(\mathrm{dd}, J=1.2,11.6 \mathrm{~Hz}, 1 \mathrm{H}), 6.50-6.62(\mathrm{~m}, 1 \mathrm{H}), 7.33-7.48(\mathrm{~m}, 10 \mathrm{H}) ;{ }^{13} \mathrm{C} \mathrm{NMR} \delta$ $61.5\left(\mathrm{~d}, J_{\mathrm{C}-\mathrm{P}}=23.4 \mathrm{~Hz}\right), 128.6\left(\mathrm{~d}, J_{\mathrm{C}-\mathrm{P}}=6.7 \mathrm{~Hz}\right), 128.7\left(\mathrm{~d}, J_{\mathrm{C}-\mathrm{P}}=6.8 \mathrm{~Hz}\right), 130.1\left(\mathrm{~d}, J_{\mathrm{C}-\mathrm{P}}=13.1\right.$ $\mathrm{Hz}), 132.6\left(\mathrm{~d}, J_{\mathrm{C}-\mathrm{P}}=18.9 \mathrm{~Hz}\right), 138.5\left(\mathrm{~d}, J_{\mathrm{C}-\mathrm{P}}=9.6 \mathrm{~Hz}\right), 144.9\left(\mathrm{~d}, J_{\mathrm{C}-\mathrm{P}}=20.5 \mathrm{~Hz}\right) ;{ }^{31} \mathrm{P}\left\{{ }^{1} \mathrm{H}\right\} \mathrm{NMR} \delta$ -29.1. HRMS calcd. for $\mathrm{C}_{15} \mathrm{H}_{16} \mathrm{O}_{2} \mathrm{P}$ (phosphine oxide) : $259.0888[\mathrm{M}+\mathrm{H}]^{+}$, found: 259.0855 . 


\section{(E)-1-Phenyl-2-diphenylphosphinyl-1-propene (3e)}

This compound was synthesized following the general procedure from 1-phenyl-1-propyne (58 mg, $0.5 \mathrm{mmol}$ ). Flash chromatography (eluent: AcOEt $/ \mathrm{n}-$ Hexane $=1 / 9, \mathrm{R}_{\mathrm{f}} 0.4$ ) yielded $103 \mathrm{mg}$ $(68 \%)$ as colorless oil. $\quad$ IR (neat) $713.6,1101.3,1636,2882 \mathrm{~cm}-1 ;{ }^{1} \mathrm{H}$ NMR $\delta 1.79$ (dd, $J=1.6$, $2.8 \mathrm{~Hz}, 3 \mathrm{H}), 7.32(\mathrm{~d}, J=1.6 \mathrm{~Hz}, 1 \mathrm{H}), 7.33-7.40(\mathrm{~m}, 15 \mathrm{H}) ;{ }^{13} \mathrm{C}$ NMR $\delta 24.4\left(\mathrm{~d}, J_{\mathrm{C}-\mathrm{P}}=4.2 \mathrm{~Hz}\right)$ $127.3,127.8,128.3\left(\mathrm{~d}, J_{\mathrm{C}-\mathrm{P}}=6.4 \mathrm{~Hz}\right), 128.4\left(\mathrm{~d}, J_{\mathrm{C}-\mathrm{P}}=6.2 \mathrm{~Hz}\right), 129.4\left(\mathrm{~d}, J_{\mathrm{C}-\mathrm{P}}=7.2\right), 133.2\left(\mathrm{~d}, J_{\mathrm{C}-\mathrm{P}}\right.$ $=18.6 \mathrm{~Hz}), 134.0,\left(\mathrm{~d}, J_{\mathrm{C}-\mathrm{P}}=21.1 \mathrm{~Hz}\right), 136.9\left(\mathrm{~d}, J_{\mathrm{C}-\mathrm{P}}=12.3 \mathrm{~Hz}\right), 137.4\left(\mathrm{~d}, \quad J_{\mathrm{C}-\mathrm{P}}=6.6 \mathrm{~Hz}\right), 143.2$ $\left(\mathrm{d}, J_{\mathrm{C}-\mathrm{P}}=29.1 \mathrm{~Hz}\right) ;{ }^{31} \mathrm{P}\left\{{ }^{1} \mathrm{H}\right\} \mathrm{NMR} \delta$-12.7. $\quad$ HRMS calcd. for $\mathrm{C}_{21} \mathrm{H}_{20} \mathrm{P}: 303.1303[\mathrm{M}+\mathrm{H}]^{+}$, found: 303.1333.

\section{(E)-6-Diphenylphosphinoyl-6-dodecene (3g)}

This compound was synthesized following the general procedure from 6-dodecyne $(83 \mathrm{mg}, 0.5$ mmol). Flash chromatography (eluent: AcOEt/n-Hexane $\left.=1 / 5, \mathrm{R}_{\mathrm{f}} 0.5\right)$ yielded $118 \mathrm{mg}(67 \%)$ as colorless oil. IR (neat) 702, 722, 1109, 1435, $3022 \mathrm{~cm}^{-1} ;{ }^{1} \mathrm{H}$ NMR $\delta 1.09-1.25$ (m, 6H), $1.26-1.34(\mathrm{~m}, 4 \mathrm{H}), 1.37-1.43(\mathrm{~m}, 2 \mathrm{H}), 2.23(\mathrm{dq}, J=3.0,7.5 \mathrm{~Hz}, 2 \mathrm{H}), 2.30-2.37(\mathrm{~m}, 2 \mathrm{H}), 6.11$ $(\mathrm{dt}, J=23.5,7.5 \mathrm{~Hz}, 1 \mathrm{H}), 7.41-7.52(\mathrm{~m}, 6 \mathrm{H}), 7.73-7.78(\mathrm{~m}, 4 \mathrm{H}) ;{ }^{13} \mathrm{C}$ NMR $\delta 13.9,14.0,22.1$, $22.4,28.1\left(\mathrm{~d}, J_{\mathrm{C}-\mathrm{P}}=11.3 \mathrm{~Hz}\right), 28.5,28.6,28.9\left(\mathrm{~d}, J_{\mathrm{C}-\mathrm{P}}=15.8 \mathrm{~Hz}\right), 29.4,29.5,31.6,32.0,128.3(\mathrm{~d}$, $\left.J_{\mathrm{C}-\mathrm{P}}=10.2 \mathrm{~Hz}\right), 131.5\left(\mathrm{~d}, J_{\mathrm{C}-\mathrm{P}}=11.5 \mathrm{~Hz}\right), 131.4\left(\mathrm{~d}, J_{\mathrm{C}-\mathrm{P}}=2.9 \mathrm{~Hz}\right), 132.4\left(\mathrm{~d}, J_{\mathrm{C}-\mathrm{P}}=80.6 \mathrm{~Hz}\right)$, $132.9\left(\mathrm{~d}, J_{\mathrm{C}-\mathrm{P}}=75.4 \mathrm{~Hz}\right), 147.3\left(\mathrm{~d}, J_{\mathrm{C}-\mathrm{P}}=10.0 \mathrm{~Hz}\right) ;{ }^{31} \mathrm{P}\left\{{ }^{1} \mathrm{H}\right\}$ NMR $\delta$ 33.2. HRMS calcd. for $\mathrm{C}_{24} \mathrm{H}_{34} \mathrm{OP}: 369.2347[\mathrm{M}+\mathrm{H}]^{+}$, found: 369.2308 . 


\section{(E)-1-Ethoxycarbonyl-2-diphenylphosphinyl-1-hexene (3h)}

This compound was synthesized following the general procedure from ethyl 2-heptynoate (77 mg, $0.5 \mathrm{mmol}$ ). Flash chromatography (eluent: AcOEt $/ \mathrm{n}-H e x a n e=1 / 3, \mathrm{R}_{\mathrm{f}} 0.4$ ) yielded $138 \mathrm{mg}$ $(81 \%)$ as colorless oil. $\quad$ IR (neat) $721,1198,1550,1733,2890 \mathrm{~cm}^{-1} ;{ }^{1} \mathrm{H}$ NMR $\delta 0.87(\mathrm{t}, J=7.2$ $\mathrm{Hz}, 3 \mathrm{H}), 1.24(\mathrm{t}, J=7.2 \mathrm{~Hz}, 3 \mathrm{H}), 1.33(\mathrm{tq}, J=7.2,7.6 \mathrm{~Hz}, 2 \mathrm{H}), 1.56-1.60(\mathrm{~m}, 2 \mathrm{H}), 2.61-2.69(\mathrm{~m}$, $2 \mathrm{H}), 4.12(\mathrm{q}, J=7.2 \mathrm{~Hz}, 2 \mathrm{H}), 5.49(\mathrm{~d}, J=6.8 \mathrm{~Hz}, 1 \mathrm{H}), 7.38-7.41(\mathrm{~m}, 10 \mathrm{H}) ; \quad{ }^{13} \mathrm{C} \mathrm{NMR} \delta 13.8$, $14.3,23.1,32.7\left(\mathrm{~d}, J_{\mathrm{C}-\mathrm{P}}=5.1 \mathrm{~Hz}\right), 33.2\left(\mathrm{~d}, J_{\mathrm{C}-\mathrm{P}}=22.1 \mathrm{~Hz}\right), 59.9,123.9\left(\mathrm{~d}, J_{\mathrm{C}-\mathrm{P}}=8.5 \mathrm{~Hz}\right), 128.8(\mathrm{~d}$, $\left.J_{\mathrm{C}-\mathrm{P}}=7.7 \mathrm{~Hz}\right), 129.5,134.0\left(\mathrm{~d}, J_{\mathrm{C}-\mathrm{P}}=9.6 \mathrm{~Hz}\right), 134.8\left(\mathrm{~d}, J_{\mathrm{C}-\mathrm{P}}=20.2 \mathrm{~Hz}\right), 164.4\left(\mathrm{~d}, J_{\mathrm{C}-\mathrm{P}}=23.7 \mathrm{~Hz}\right)$, $165.2\left(\mathrm{~d}, J_{\mathrm{C}-\mathrm{P}}=7.5 \mathrm{~Hz}\right) ;{ }^{31} \mathrm{P}\left\{{ }^{1} \mathrm{H}\right\} \mathrm{NMR} \delta$ 1.75. HRMS calcd. for $\mathrm{C}_{21} \mathrm{H}_{26} \mathrm{O}_{2} \mathrm{P}: 341.1670[\mathrm{M}+\mathrm{H}]^{+}$, found: 341.1642 .

\section{(E)- $\alpha$-Diphenylphosphinoyl- $\beta$-Ethoxycarbonylstyrene (3i)}

This compound was synthesized following the general procedure from ethyl phenylpropiolate (87 mg, $0.5 \mathrm{mmol})$. Flash chromatography (eluent: AcOEt $/ \mathrm{n}-\mathrm{Hexane}=1 / 3, \mathrm{R}_{\mathrm{f}} 0.3$ ) yielded 143 mg $\left(76 \%\right.$ ) as colorless oil. IR (neat) $801,1102,1450,1770 \mathrm{~cm}^{-1}$; (major isomer) ${ }^{1} \mathrm{H}$ NMR $\delta$ $0.98(\mathrm{t}, J=7.2 \mathrm{~Hz}, 3 \mathrm{H}), 3.96(\mathrm{q}, J=7.2 \mathrm{~Hz}, 2 \mathrm{H}), 6.79(\mathrm{~d}, J=19.2 \mathrm{~Hz}, 1 \mathrm{H}), 7.12-7.37(\mathrm{~m}, 5 \mathrm{H})$, 7.37-7.65 (m, 10H); ${ }^{13} \mathrm{C}$ NMR $\delta 13.8,60.8,127.9,128.0\left(\mathrm{~d}, J_{\mathrm{C}-\mathrm{P}}=12.3 \mathrm{~Hz}\right), 128.1,128.5\left(\mathrm{~d}, J_{\mathrm{C}-\mathrm{P}}\right.$ $=12.3 \mathrm{~Hz}), 130.1,131.9\left(\mathrm{~d}, J_{\mathrm{C}-\mathrm{P}}=10.0 \mathrm{~Hz}\right), 132.2\left(\mathrm{~d}, J_{\mathrm{C}-\mathrm{P}}=9.6 \mathrm{~Hz}\right), 133.1\left(\mathrm{~d}, J_{\mathrm{C}-\mathrm{P}}=10.6 \mathrm{~Hz}\right)$, $136.0\left(\mathrm{~d}, J_{\mathrm{C}-\mathrm{P}}=5.7 \mathrm{~Hz}\right), 149.5\left(\mathrm{~d}, J_{\mathrm{C}-\mathrm{P}}=85.0 \mathrm{~Hz}\right), 164.8\left(\mathrm{~d}, J_{\mathrm{C}-\mathrm{P}}=22.0 \mathrm{~Hz}\right) ;{ }^{31} \mathrm{P}\left\{{ }^{1} \mathrm{H}\right\} \mathrm{NMR} \delta 27.9$. HRMS calcd. for $\mathrm{C}_{23} \mathrm{H}_{22} \mathrm{O}_{3} \mathrm{P}$ (phosphine oxide): $377.1306[\mathrm{M}+\mathrm{H}]^{+}$, found: 377.1303 .

(minor isomer) ${ }^{1} \mathrm{H}$ NMR $\delta 1.08(\mathrm{t}, J=6.8 \mathrm{~Hz}, 3 \mathrm{H}), 3.96(\mathrm{q}, J=6.8 \mathrm{~Hz}, 2 \mathrm{H}), 6.71(\mathrm{~d}, J=32.8 \mathrm{~Hz}$, 
$1 \mathrm{H}), 7.12-7.37(\mathrm{~m}, 5 \mathrm{H}), 7.37-7.65(\mathrm{~m}, 10 \mathrm{H}) ;{ }^{13} \mathrm{C}$ NMR $\delta 13.7,61.4,127.8,128.1,128.4\left(\mathrm{~d}, J_{\mathrm{C}-\mathrm{P}}=\right.$ $10.3 \mathrm{~Hz}), 128.7\left(\mathrm{~d}, J_{\mathrm{C}-\mathrm{P}}=15.5 \mathrm{~Hz}\right), 129.1,131.5,131.8\left(\mathrm{~d}, J_{\mathrm{C}-\mathrm{P}}=8.9 \mathrm{~Hz}\right), 134.3\left(\mathrm{~d}, J_{\mathrm{C}-\mathrm{P}}=7.3 \mathrm{~Hz}\right)$, $138.1\left(\mathrm{~d}, J_{\mathrm{C}-\mathrm{P}}=8.6 \mathrm{~Hz}\right), 146.0\left(\mathrm{~d}, J_{\mathrm{C}-\mathrm{P}}=85.3 \mathrm{~Hz}\right), 165.3\left(\mathrm{~d}, J_{\mathrm{C}-\mathrm{P}}=6.4 \mathrm{~Hz}\right) ; \quad{ }^{31} \mathrm{P}\left\{{ }^{1} \mathrm{H}\right\} \mathrm{NMR} \delta$ 26.8 .

\section{(2-Ethoxycarbonylethyl)diphenylphosphane (5)}

This compound was synthesized following the general procedure from ethyl acrylate ( $30 \mathrm{mg}, 0.3$ mmol). Flash chromatography (eluent: $\left.\mathrm{CHCl}_{3} / \mathrm{n}-\mathrm{Hexane}=1 / 1, \mathrm{R}_{\mathrm{f}} 0.4\right)$ yielded $64 \mathrm{mg}(74 \%)$ as colorless oil. IR (neat) 2995, 2940, 1750, 1460, 1385, 1210, 1035, $940 \mathrm{~cm}^{-1} ; \quad{ }^{1} \mathrm{H}$ NMR $\delta 1.23$ (t, $J=7.6 \mathrm{~Hz}, 3 \mathrm{H}), 2.33-2.43(\mathrm{~m}, 4 \mathrm{H}), 4.10(\mathrm{q}, J=7.6 \mathrm{~Hz}, 2 \mathrm{H}), 7.30-7.36(\mathrm{~m}, 5 \mathrm{H}), 7.41-7.45$ $(\mathrm{m}, 5 \mathrm{H}) ;{ }^{13} \mathrm{C}$ NMR $\delta 13.6,25.2\left(\mathrm{~d}, J_{\mathrm{C}-\mathrm{P}}=14.4 \mathrm{~Hz}\right), 29.2\left(\mathrm{~d}, J_{\mathrm{C}-\mathrm{P}}=23.4 \mathrm{~Hz}\right), 59.5,128.2,128.5$, 132.7, 138.5 142.6, 172.3; ${ }^{31} \mathrm{P}\left\{{ }^{1} \mathrm{H}\right\}$ NMR $\delta$-14.4. HRMS calcd. for $\mathrm{C}_{17} \mathrm{H}_{19} \mathrm{O}_{2} \mathrm{P}: 287.1123$ $[\mathrm{M}+\mathrm{H}]^{+}$, found: 287.1138 .

\section{(2-Cyanoethyl)diphenylphosphane (7)}

This compound was synthesized following the general procedure from acrylonitrile (16 mg, 0.3 mmol). Flash chromatography (eluent: AcOEt/n-Hexane $\left.=1 / 10, R_{\mathrm{f}} 0.5\right)$ yielded $56 \mathrm{mg}(78 \%)$ as colorless oil. IR (neat) 2962, 2930, 1733, 1479, 1387, 1291, 1009, $753 \mathrm{~cm}^{-1} ;{ }^{1} \mathrm{H}$ NMR $\delta$ 7.44-7.25 (m, $10 \mathrm{H}), 2.37(\mathrm{~m}, 2 \mathrm{H}), 2.34(\mathrm{~m}, 2 \mathrm{H}) ;{ }^{13} \mathrm{C}$ NMR $\delta 14.1\left(\mathrm{~d}, J_{\mathrm{C}-\mathrm{P}}=23.7 \mathrm{~Hz}\right), 24.2(\mathrm{~d}$, $\left.J_{\mathrm{C}-\mathrm{P}}=15.6 \mathrm{~Hz}\right), 120.0,128.8,129.4,132.5,132.8,136.3 ;{ }^{31} \mathrm{P}\left\{{ }^{1} \mathrm{H}\right\} \mathrm{NMR} \delta-15.4 . \quad$ HRMS calcd. for $\mathrm{C}_{15} \mathrm{H}_{14} \mathrm{NP}: 240.0897[\mathrm{M}+\mathrm{H}]^{+}$, found: 240.0881 . 


\section{References}

1. M. Hayashi, Y. Matsuura, Y. Watanabe Tetrahedron Lett., 2004, 45, 1409-1411.

2. G. Giordano, R. H. Crabtree Inorg. Synth., 1990, 28, 88.

3. M. Taillefer, H. J. Cristau Tetrahedron Lett., 1998, 39, 7857-7860.

4. M. Hayashi, Y. Matsuura, Y. Watanabe Tetrahedron Lett., 2004, 45, 9167-9169.

5. H. Ohmiya, H. Yorimitsu, K. Oshima Angew. Chem. Int. Ed. 2005, 44, 2368-2370. 
3a $\left({ }^{1} \mathrm{H}\right)$

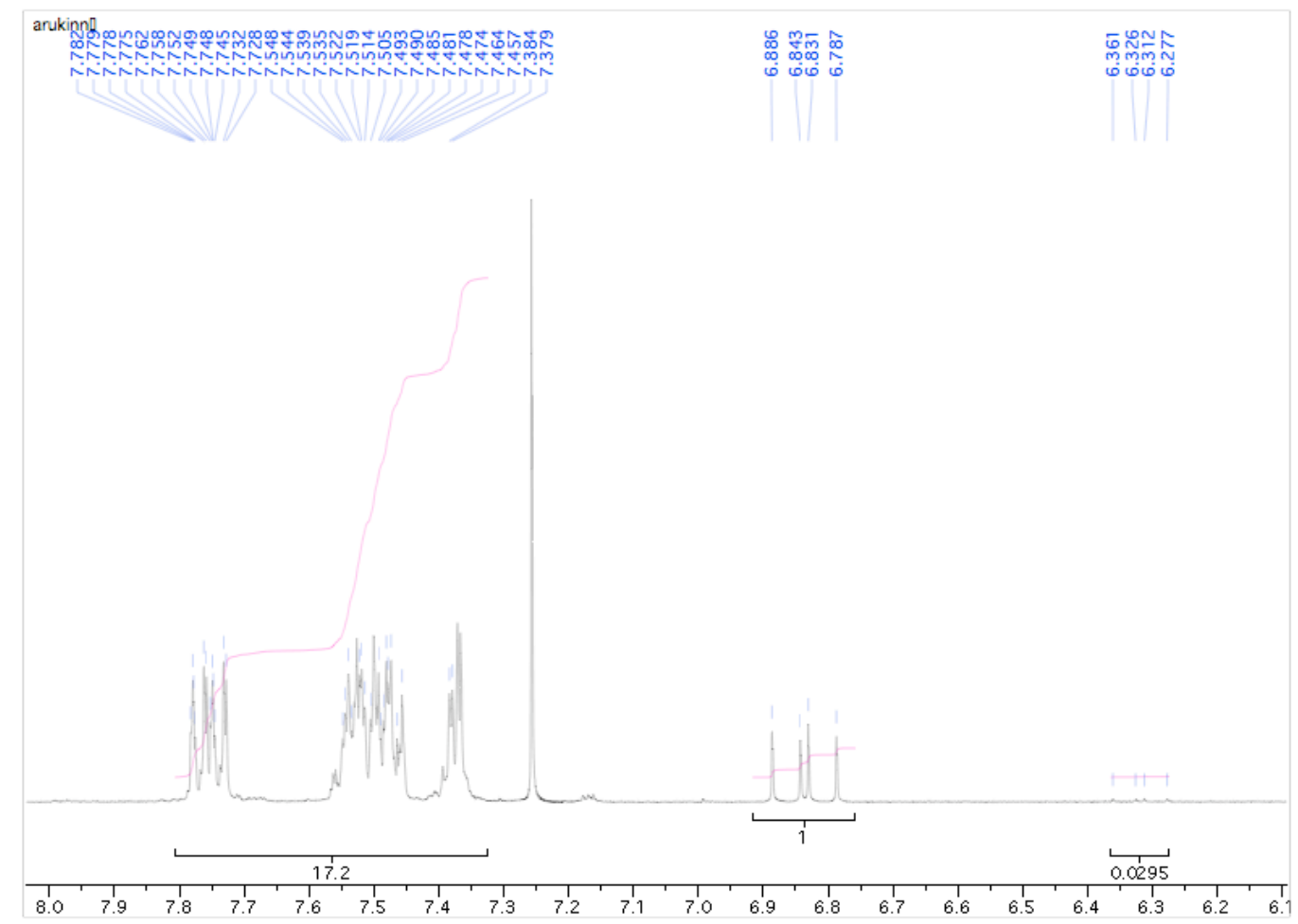

$3 a\left({ }^{13} \mathrm{C}\right)$

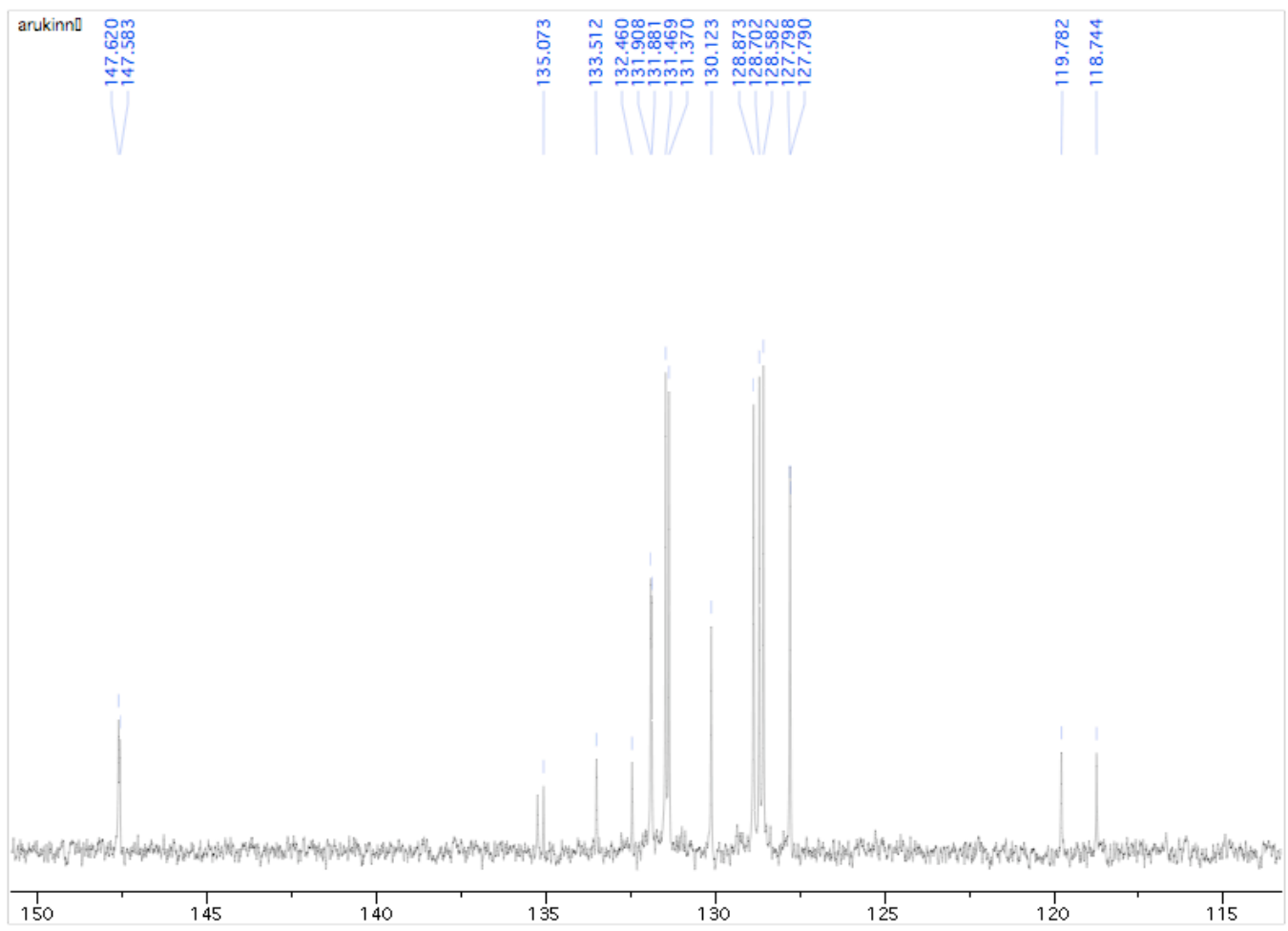


3a $\left({ }^{31} \mathrm{P}\right)$

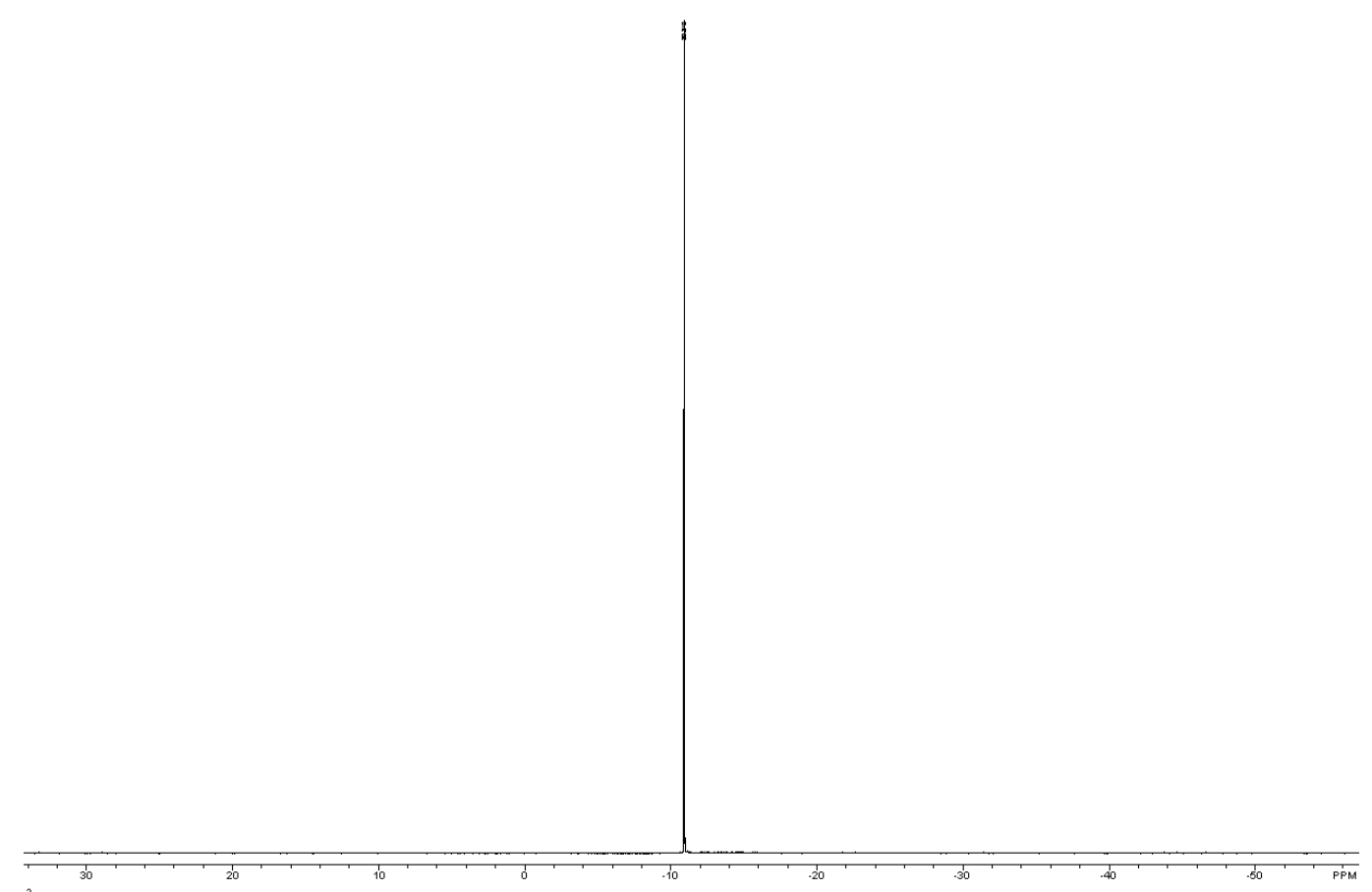

3b $\left({ }^{1} H\right)$

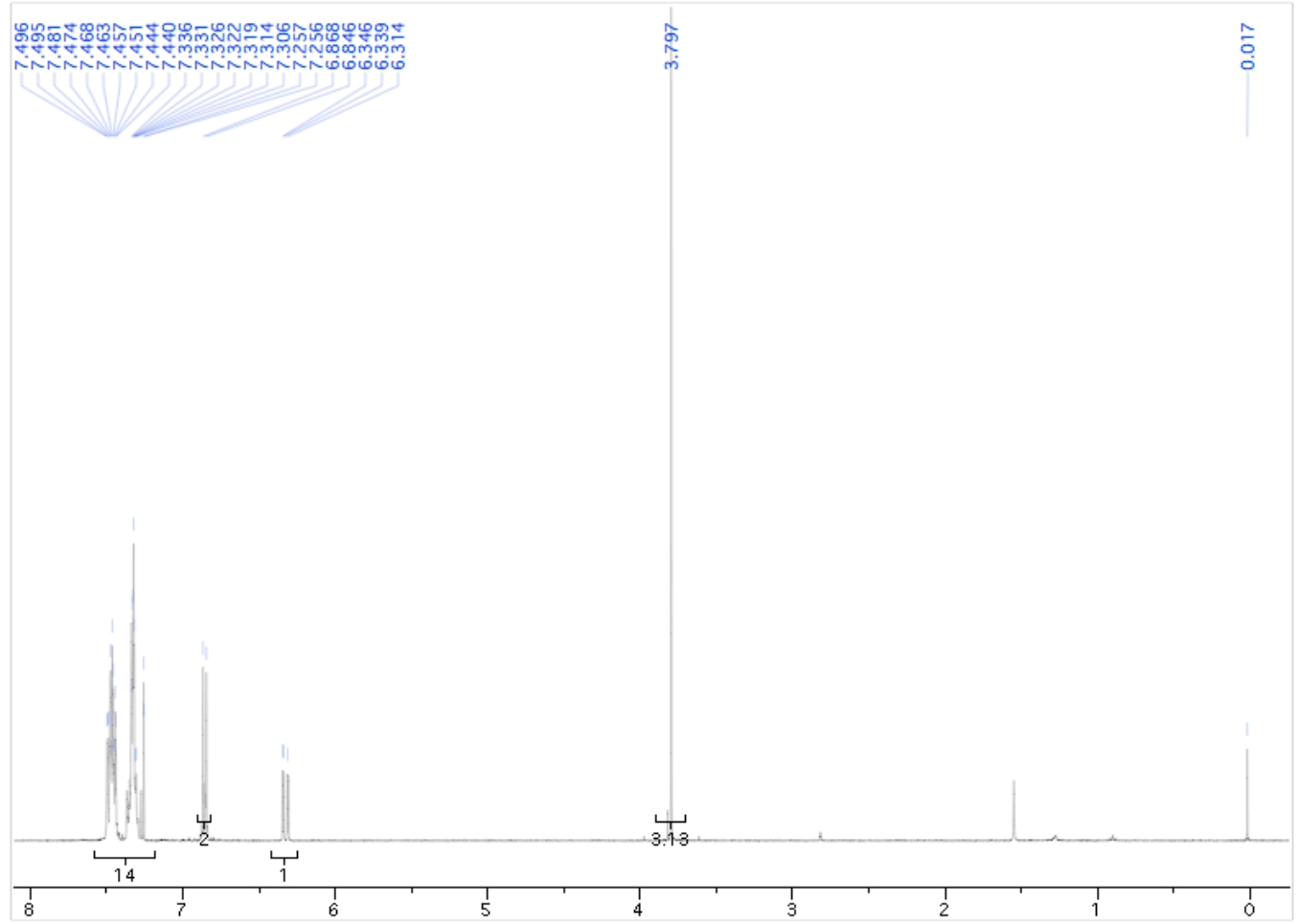

S10 
$3 b\left({ }^{13} \mathrm{C}\right)$

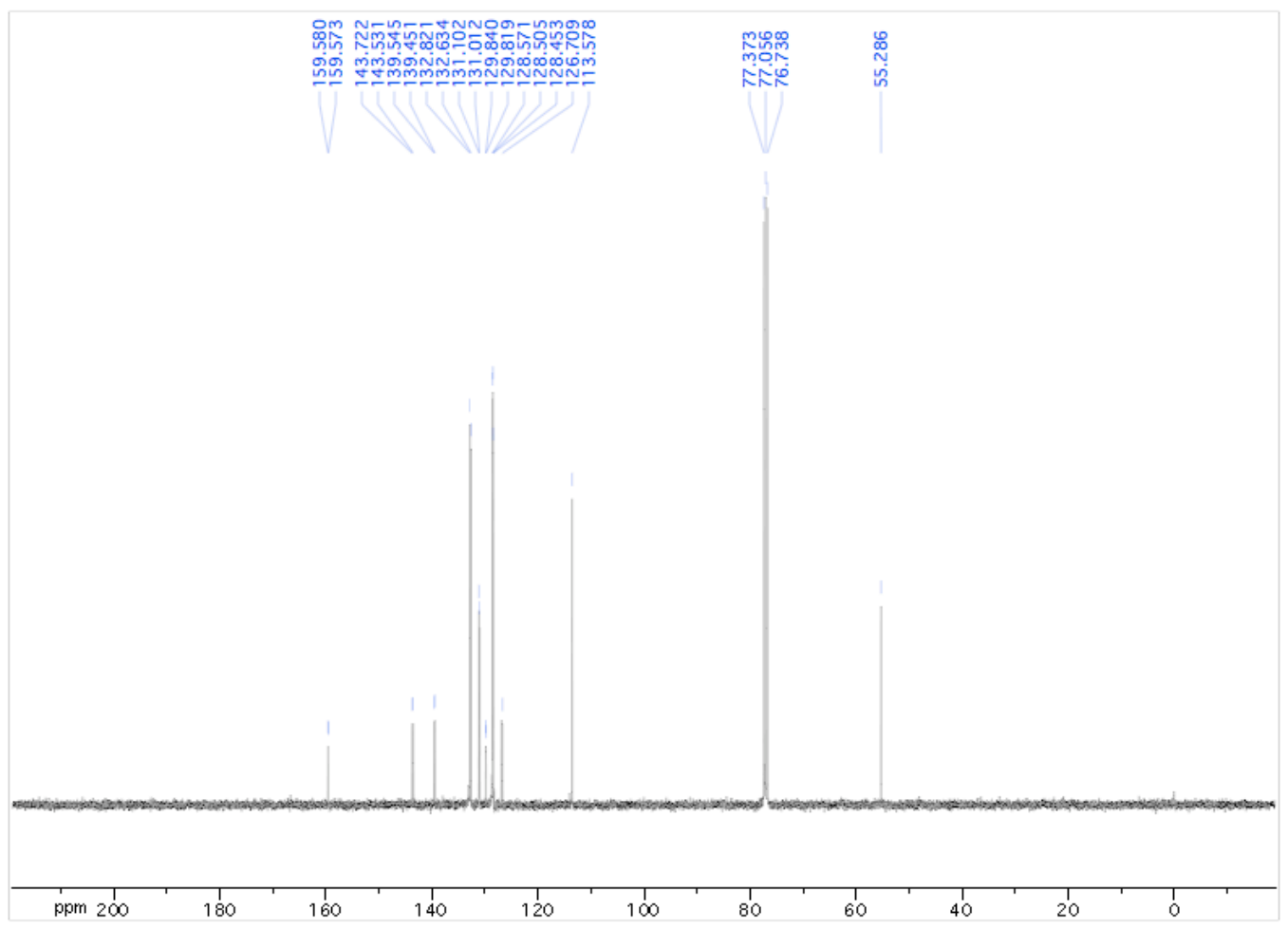

$3 b\left({ }^{31} \mathrm{P}\right)$

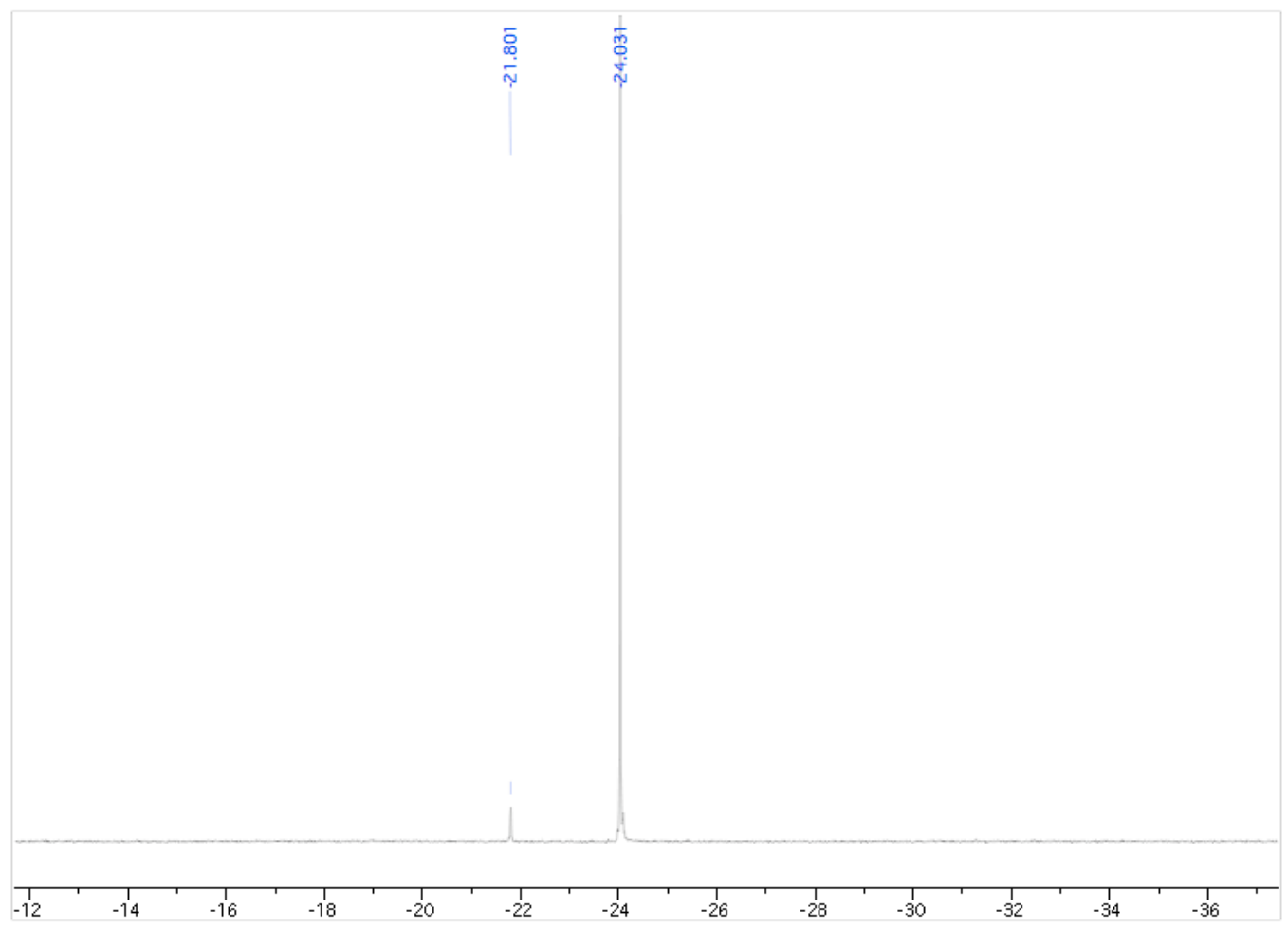


$3 c\left({ }^{1} \mathrm{H}\right)$

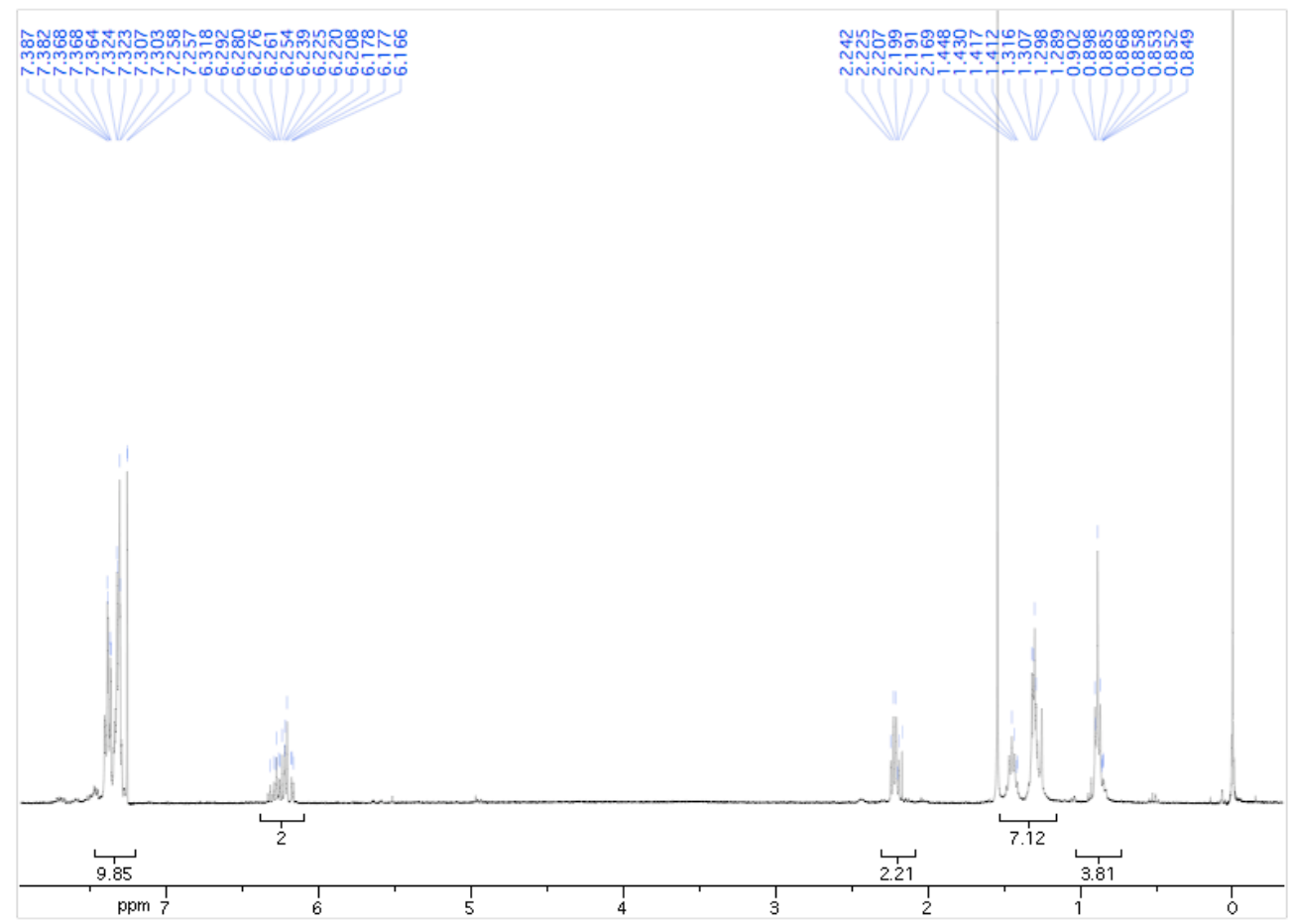

$3 c\left({ }^{13} \mathrm{C}\right)$

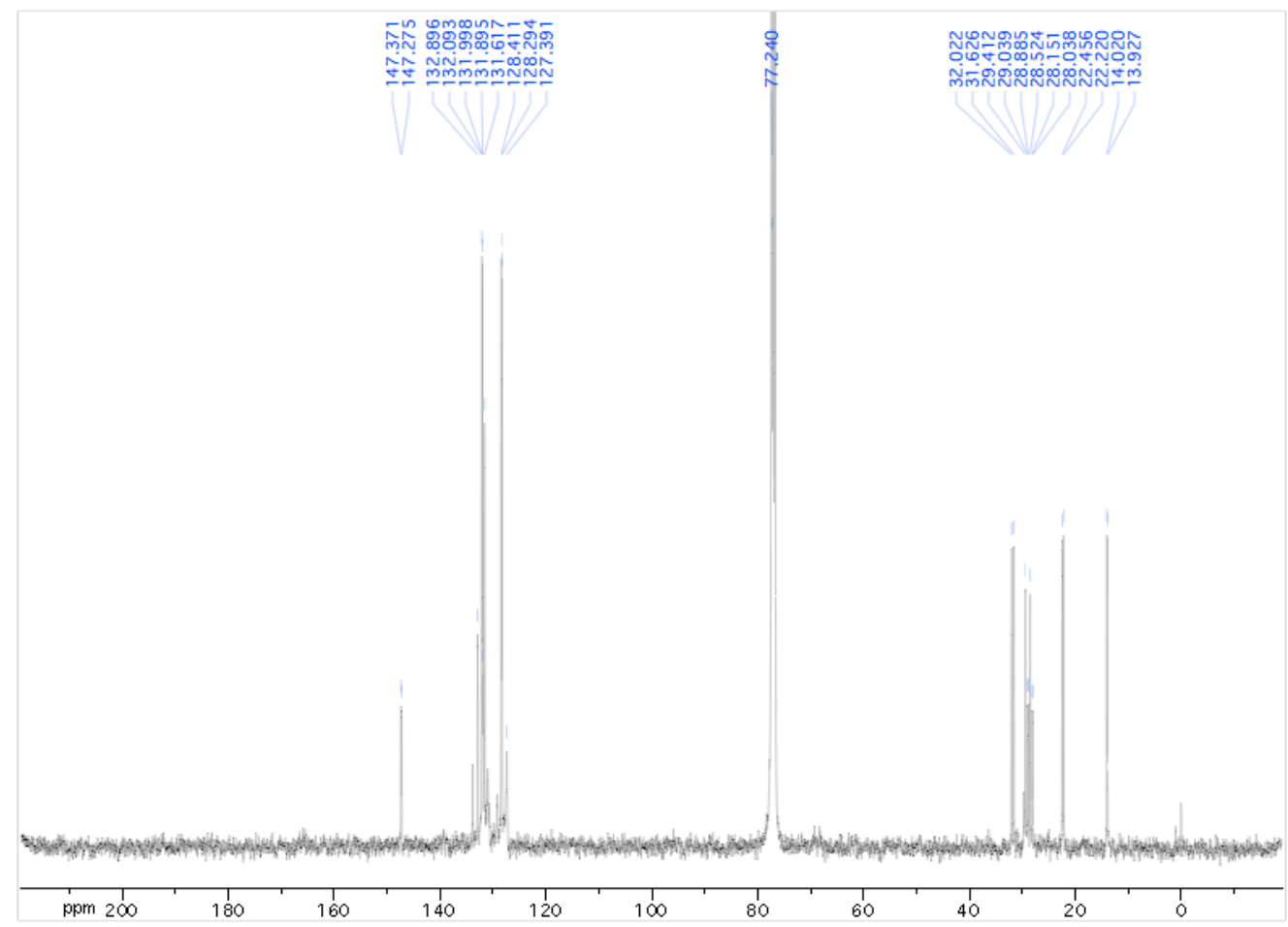


$3 c\left({ }^{31} \mathrm{P}\right)$

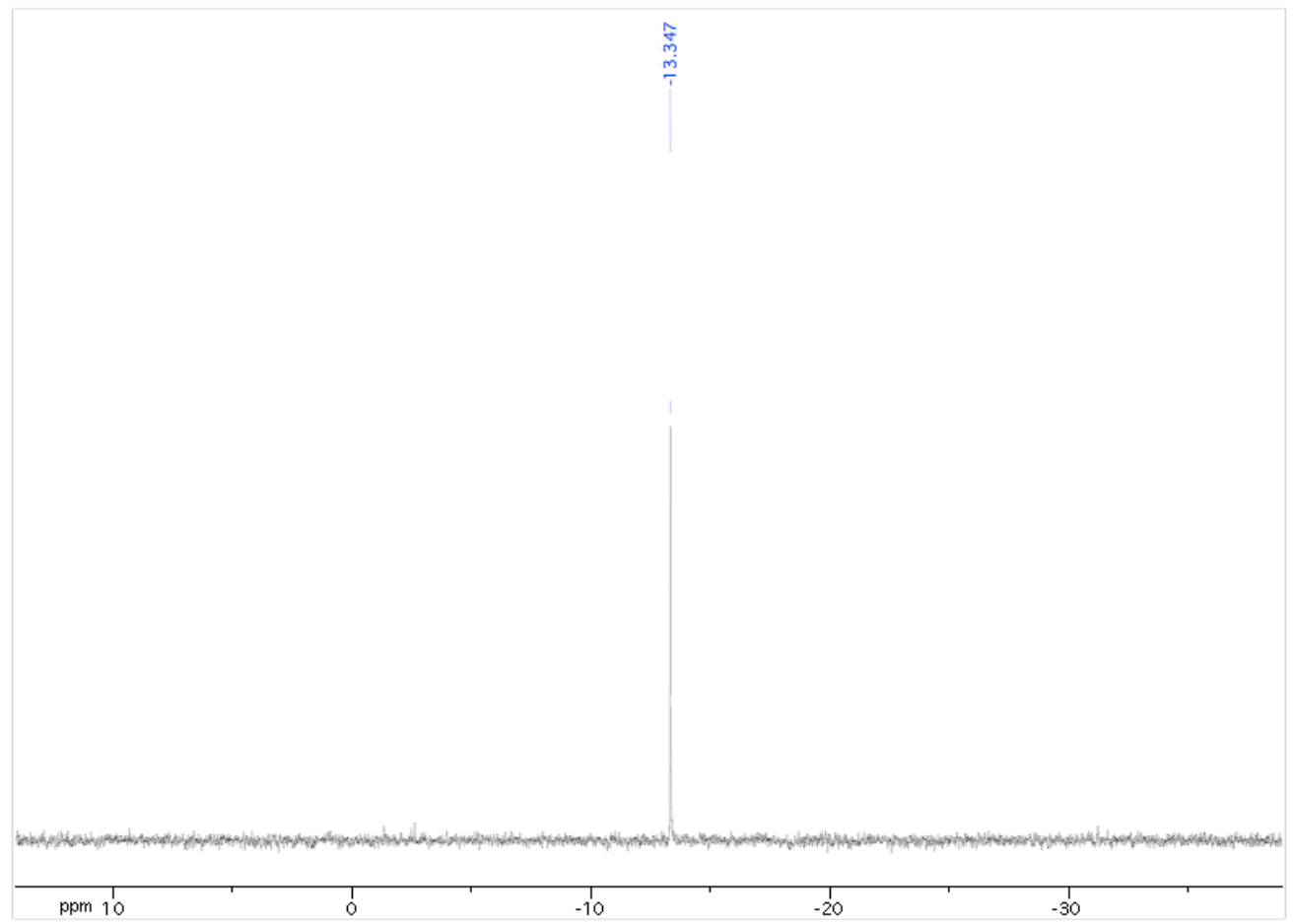

3d $\left({ }^{1} \mathrm{H}\right)$

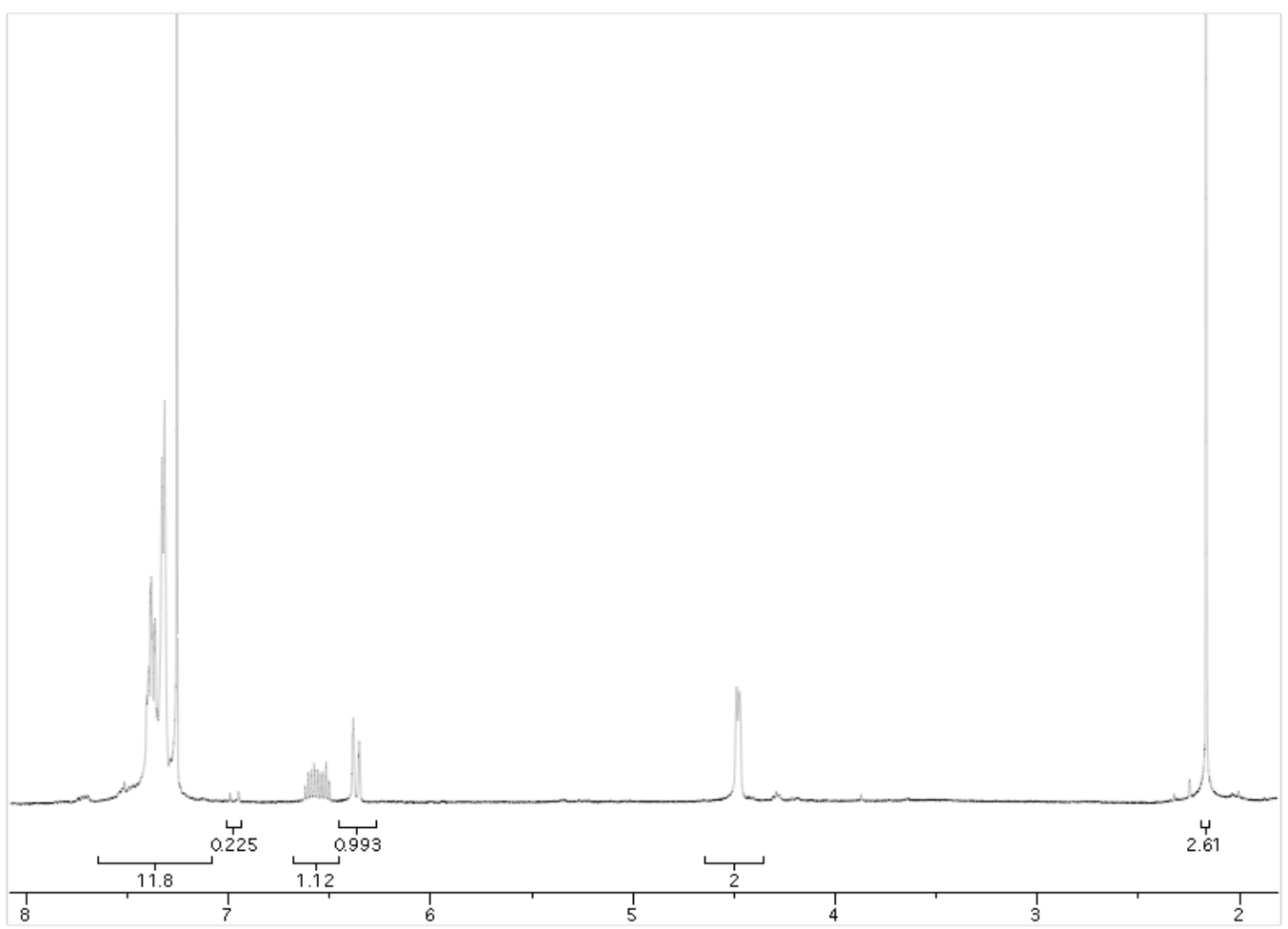

S13 


\section{3d $\left({ }^{31} \mathrm{P}\right)$}

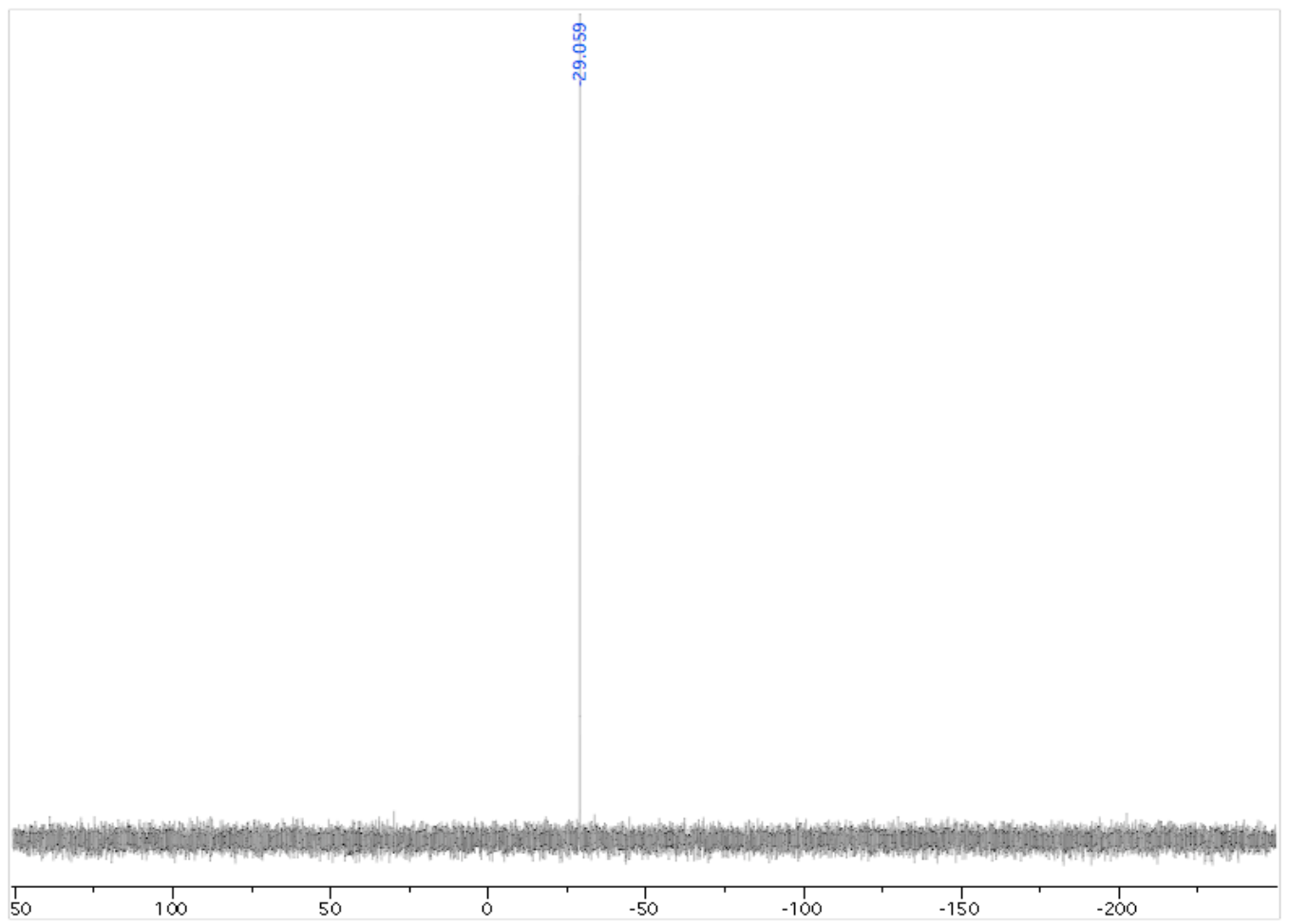

$3 d\left({ }^{13} \mathrm{C}\right)$

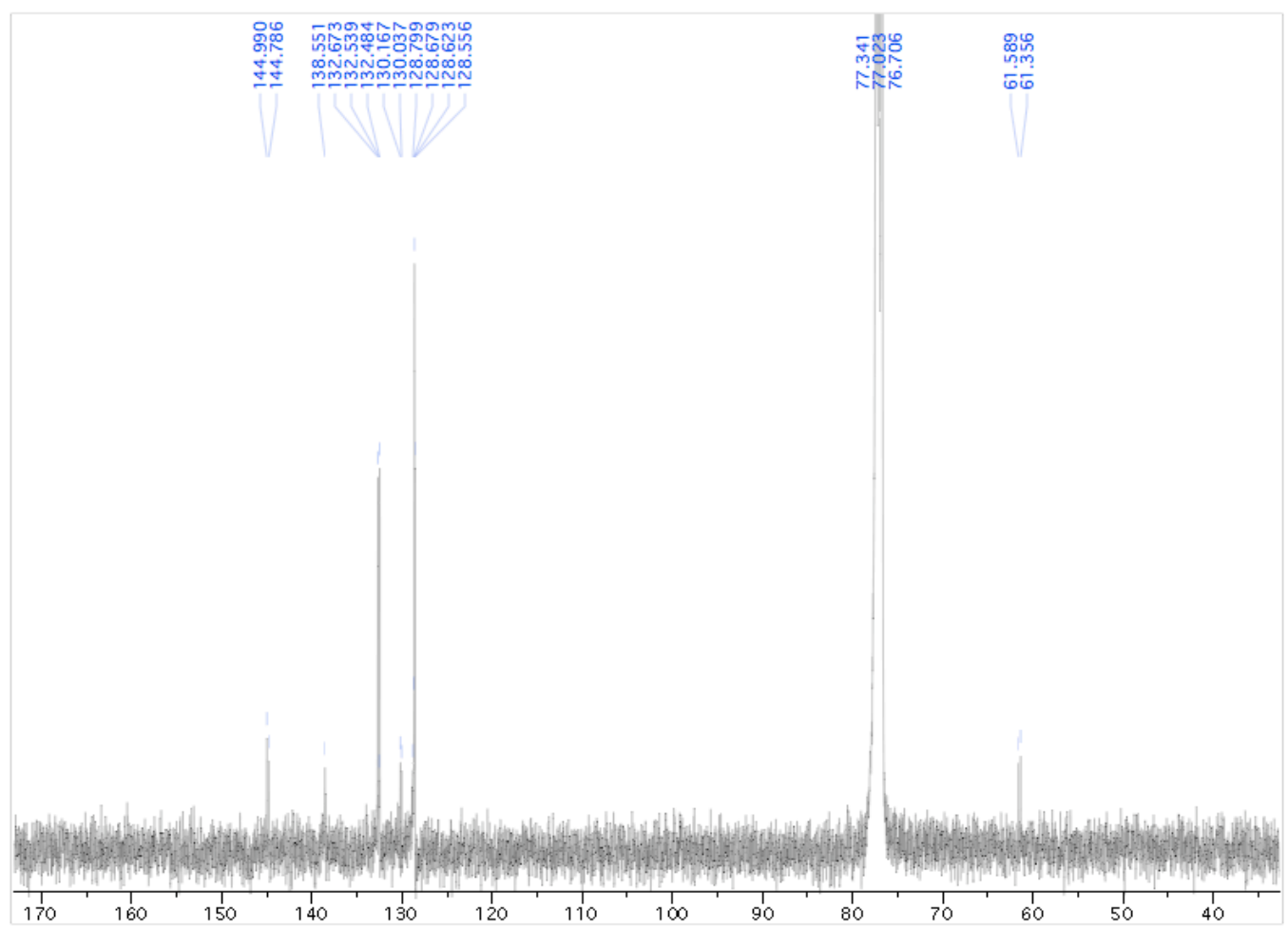


3e $\left({ }^{1} \mathrm{H}\right)$

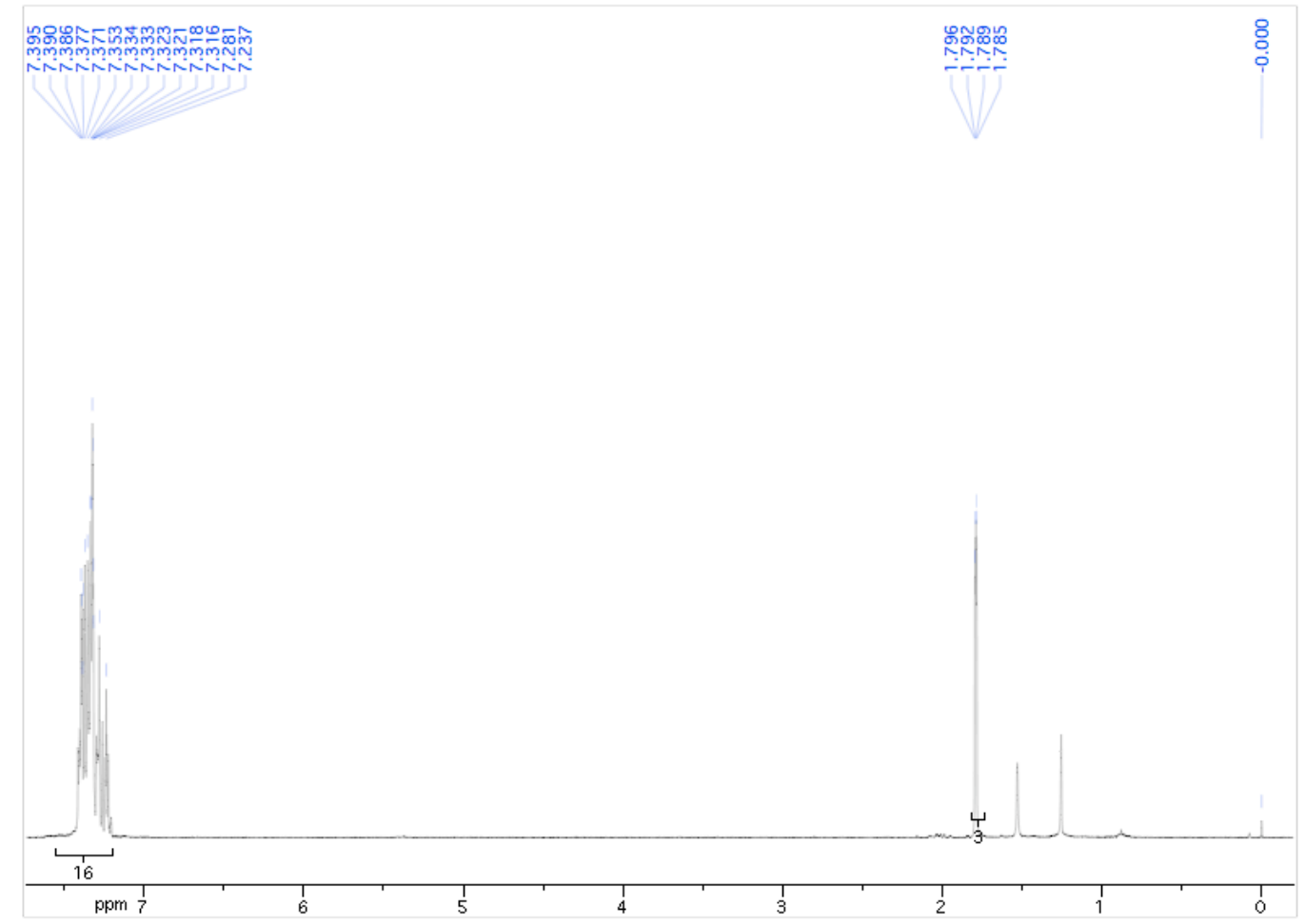

$3 e\left({ }^{13} \mathrm{C}\right)$

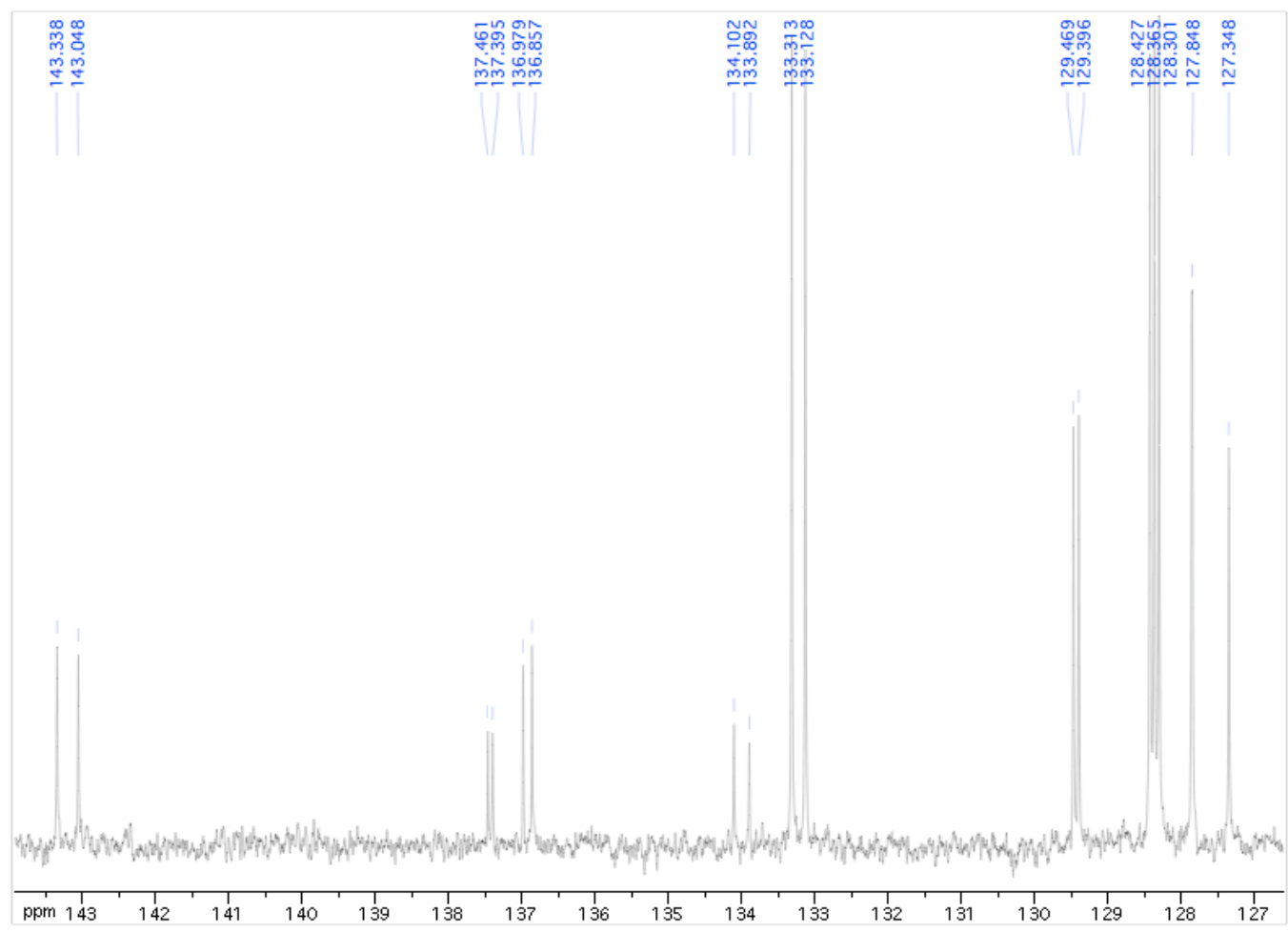




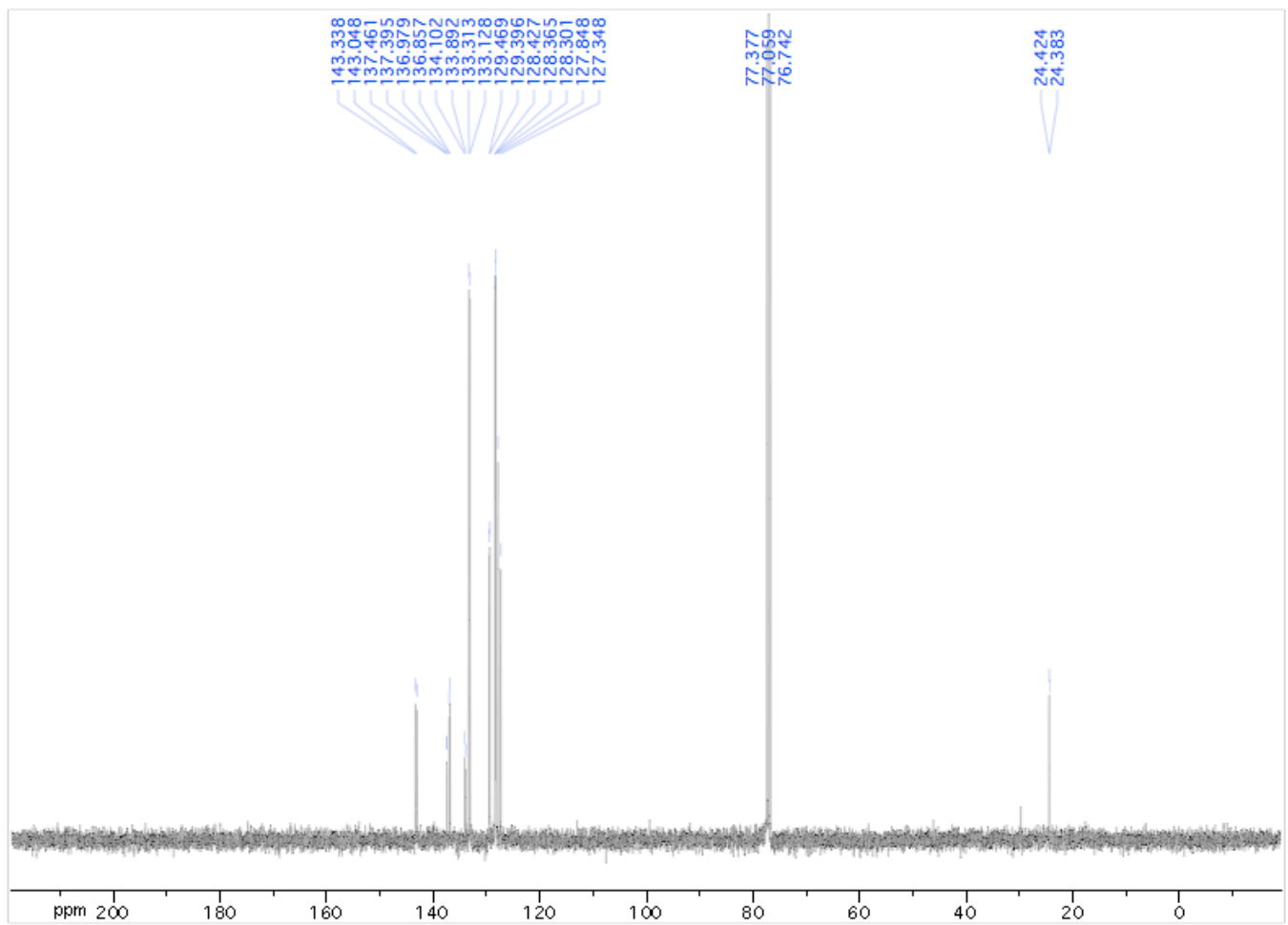

$3 e\left({ }^{31} \mathrm{P}\right)$

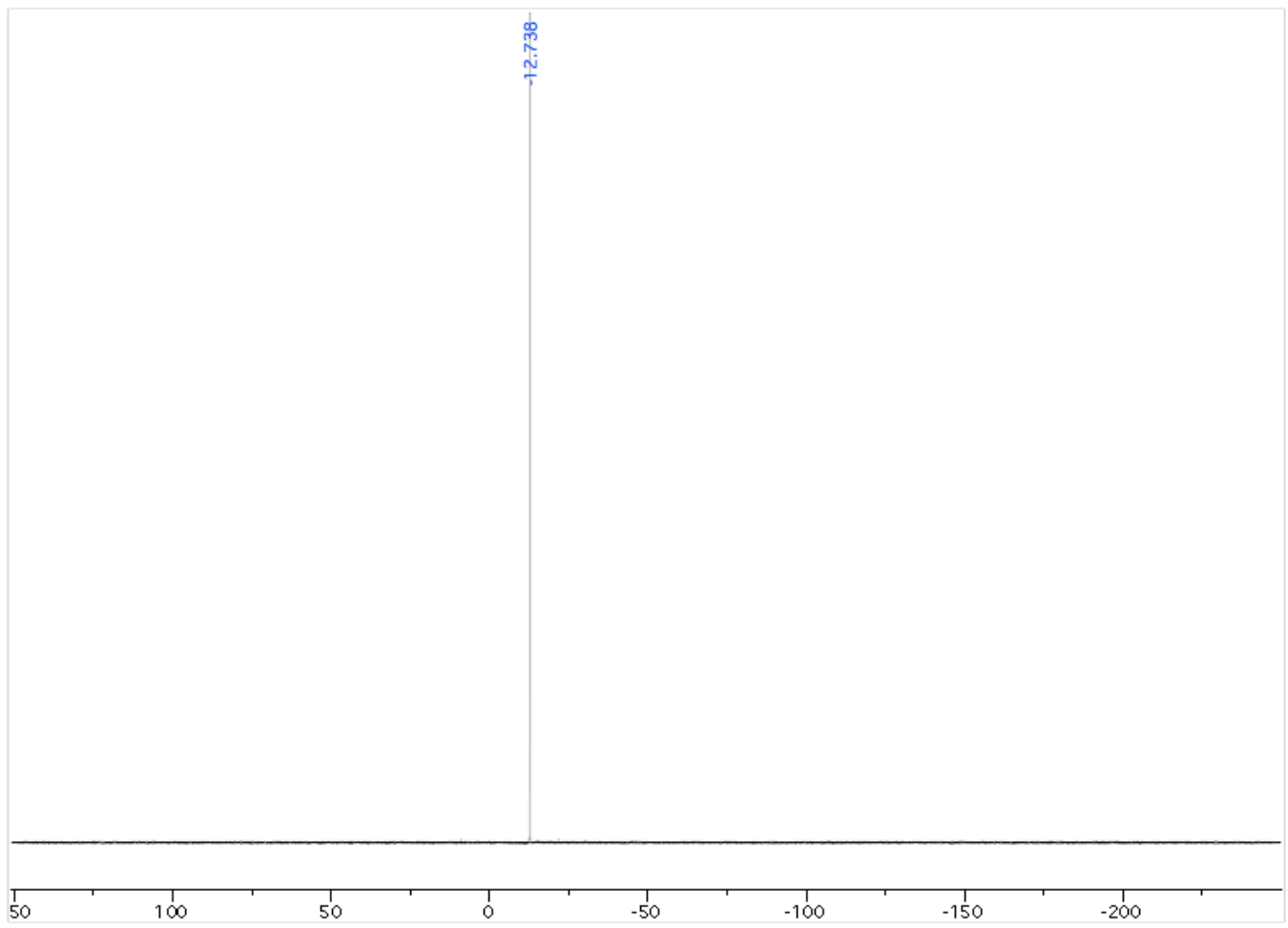

S16 
$\mathbf{3 g}\left({ }^{1} \mathrm{H}\right)$

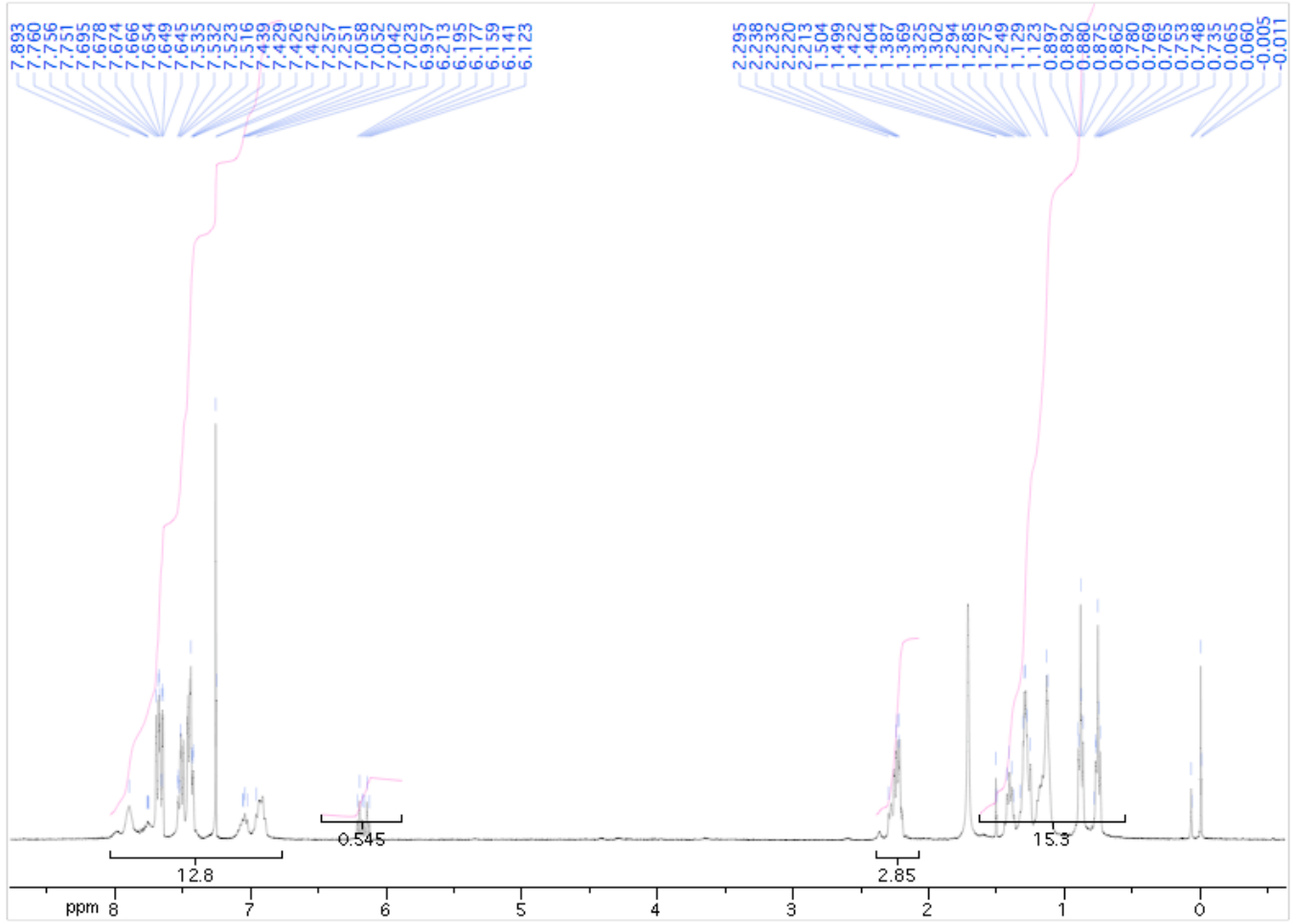

$3 g\left({ }^{13} \mathrm{C}\right)$

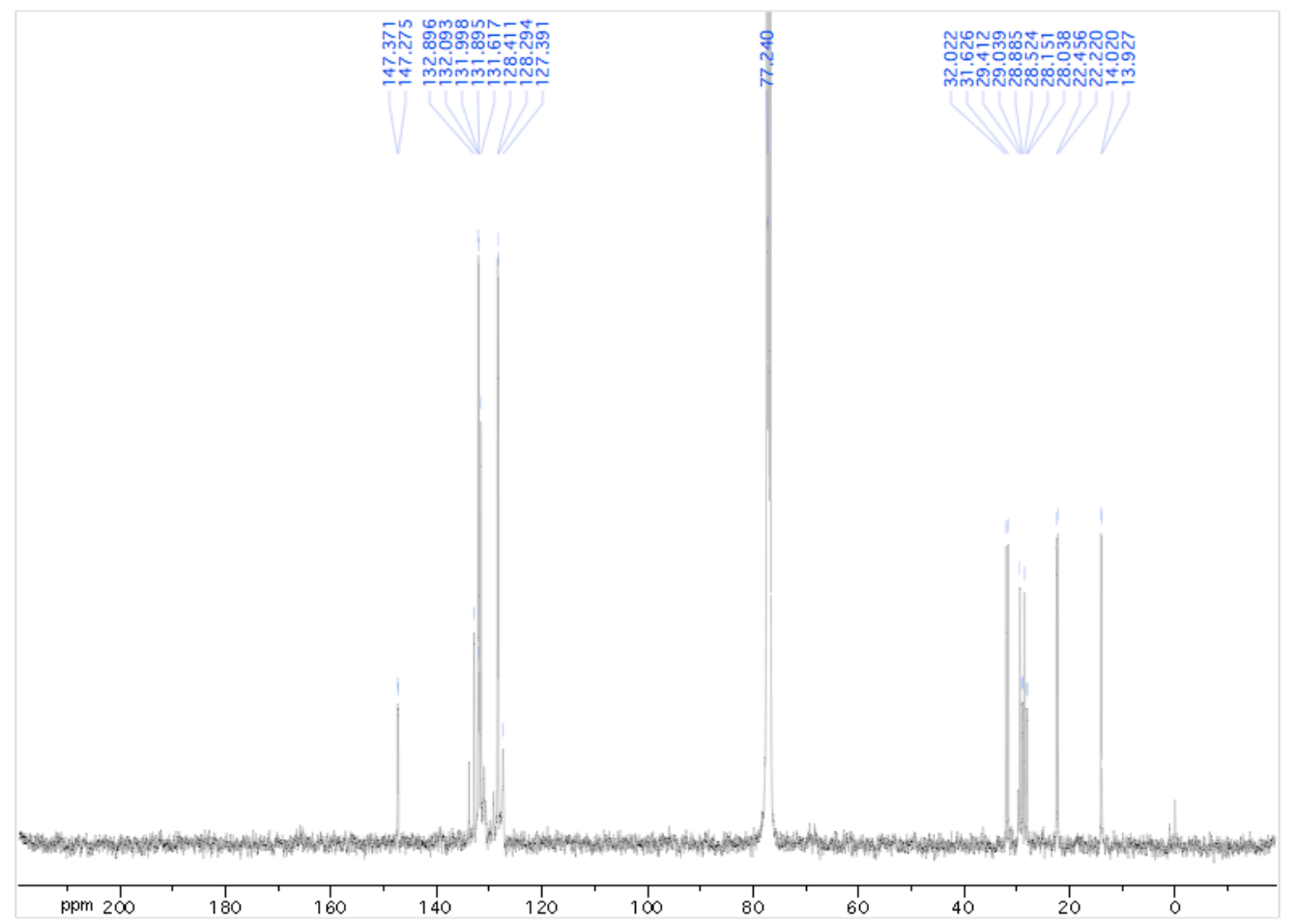


$3 g\left({ }^{31} \mathrm{P}\right)$

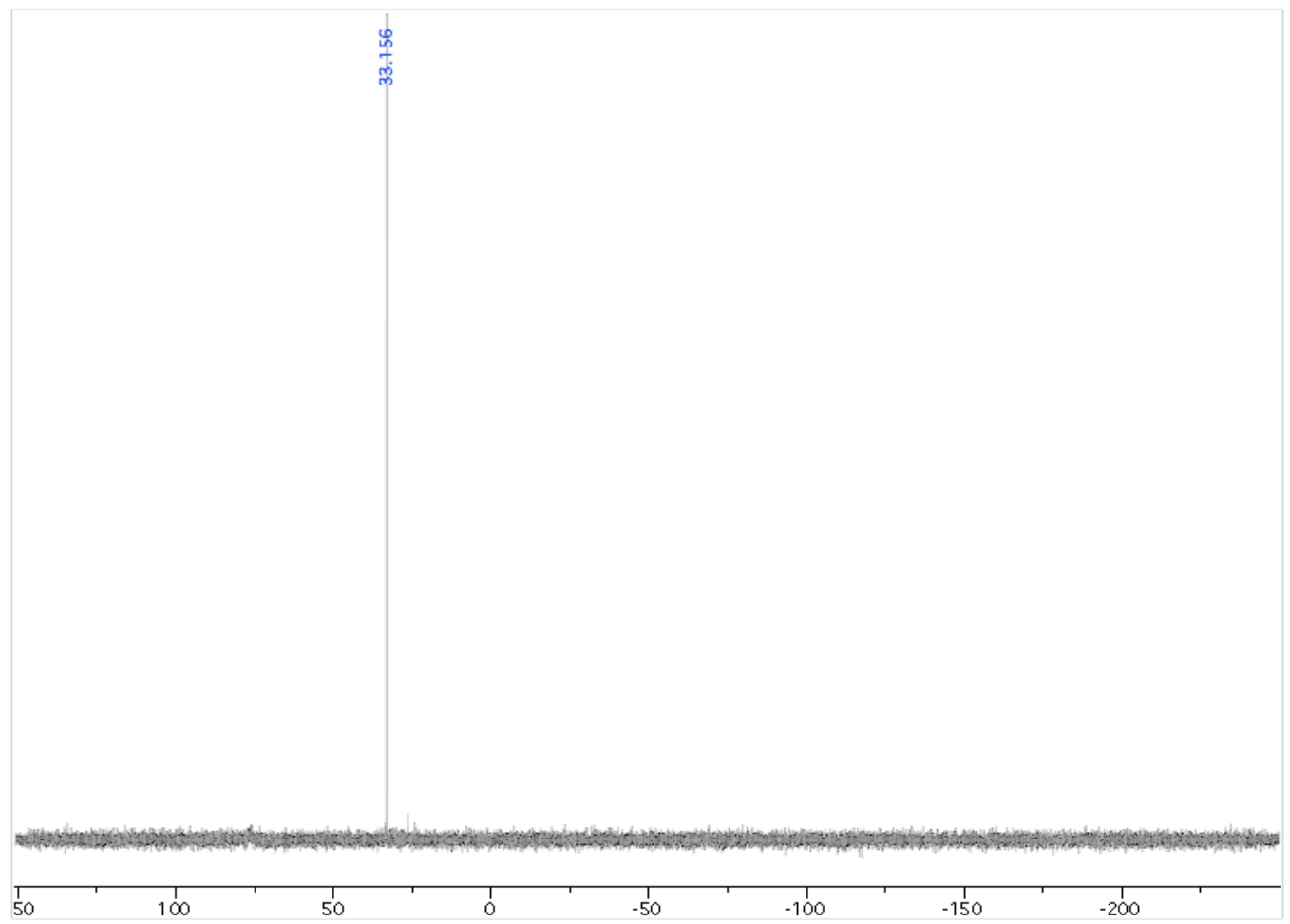

3h $\left({ }^{1} \mathrm{H}\right)$

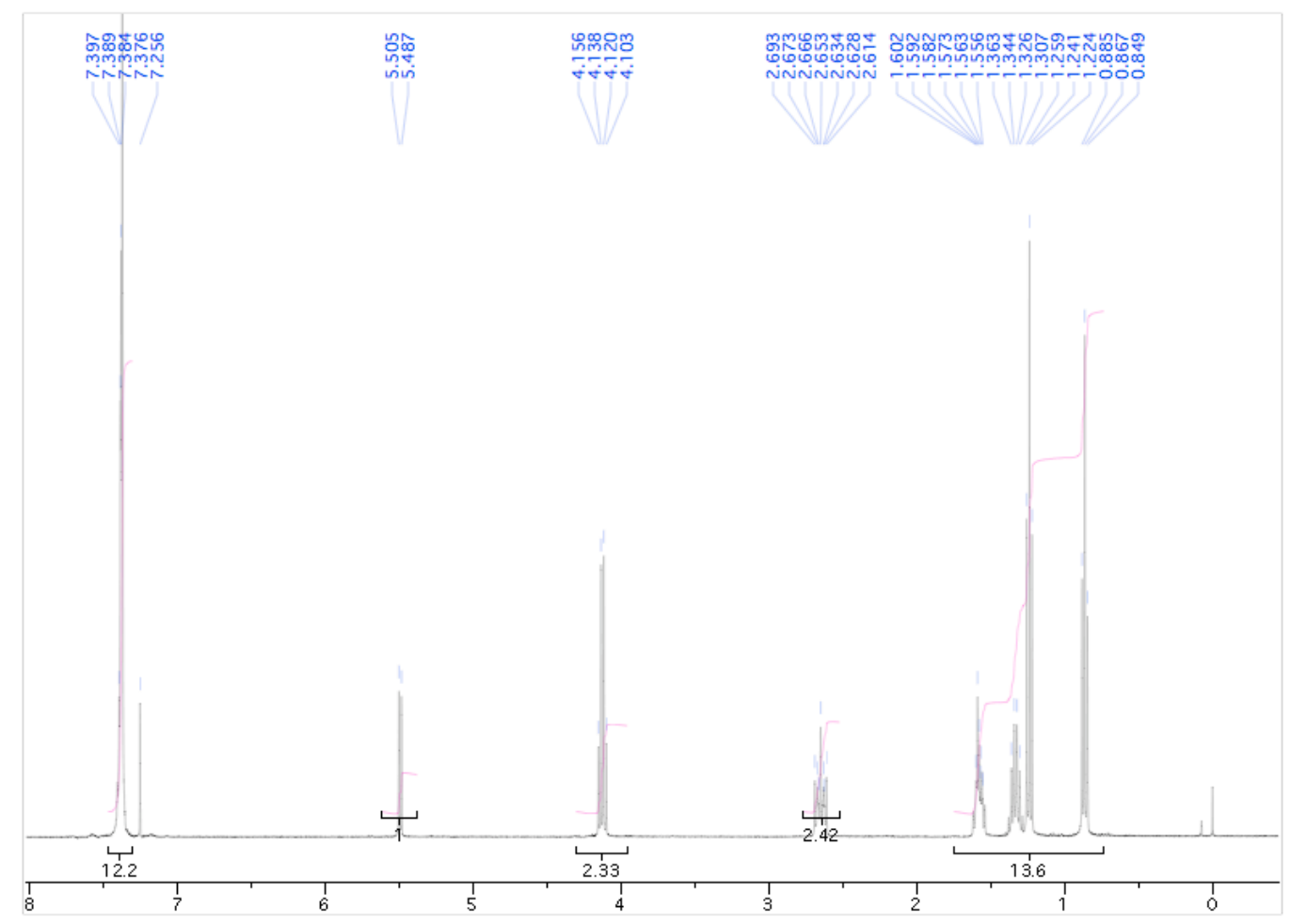

S18 
$3 h\left({ }^{13} \mathrm{C}\right)$

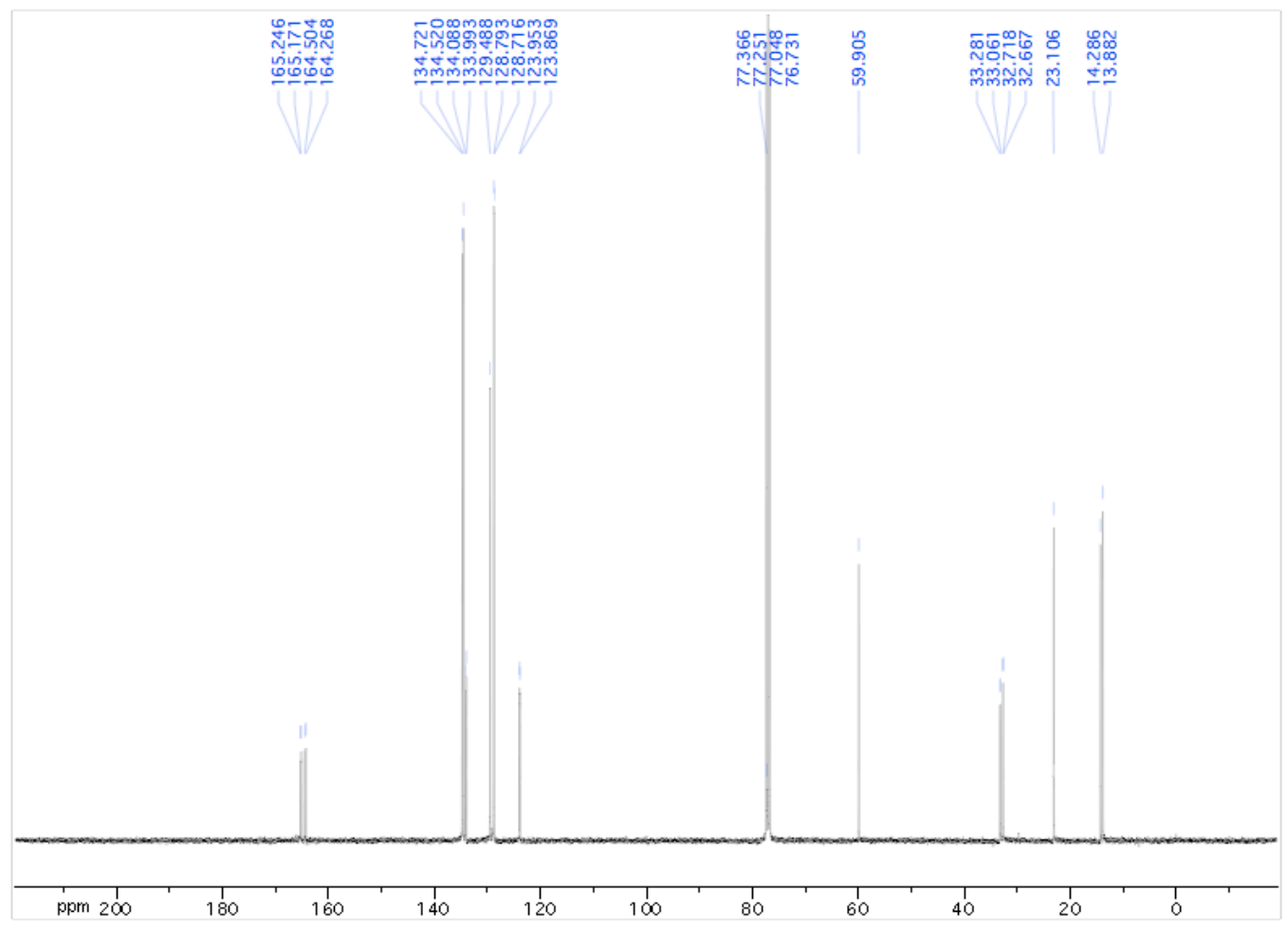

$3 \mathbf{i}\left({ }^{31} \mathrm{P}\right)$

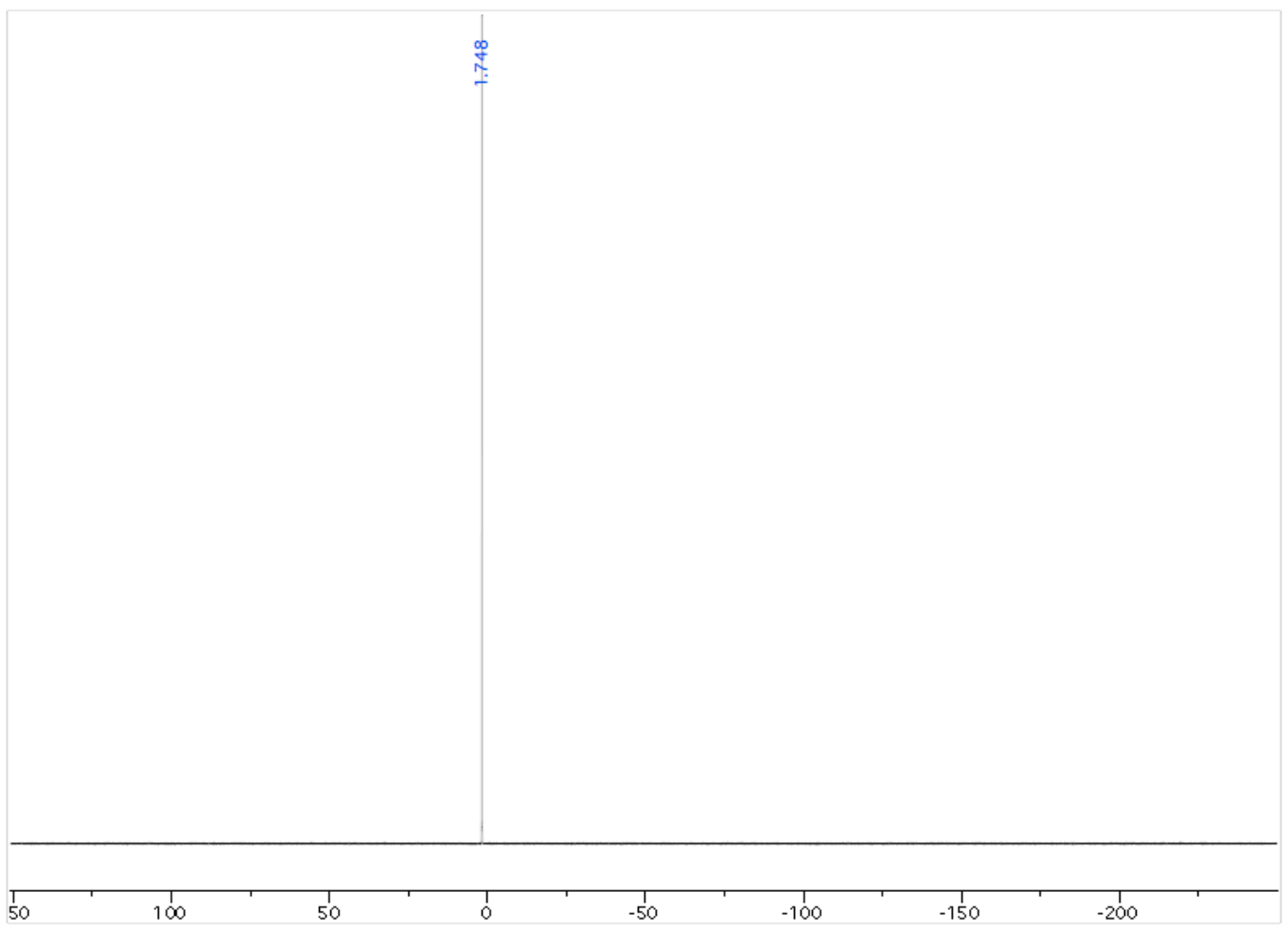


$3 \mathbf{i}\left({ }^{1} \mathrm{H}\right)$
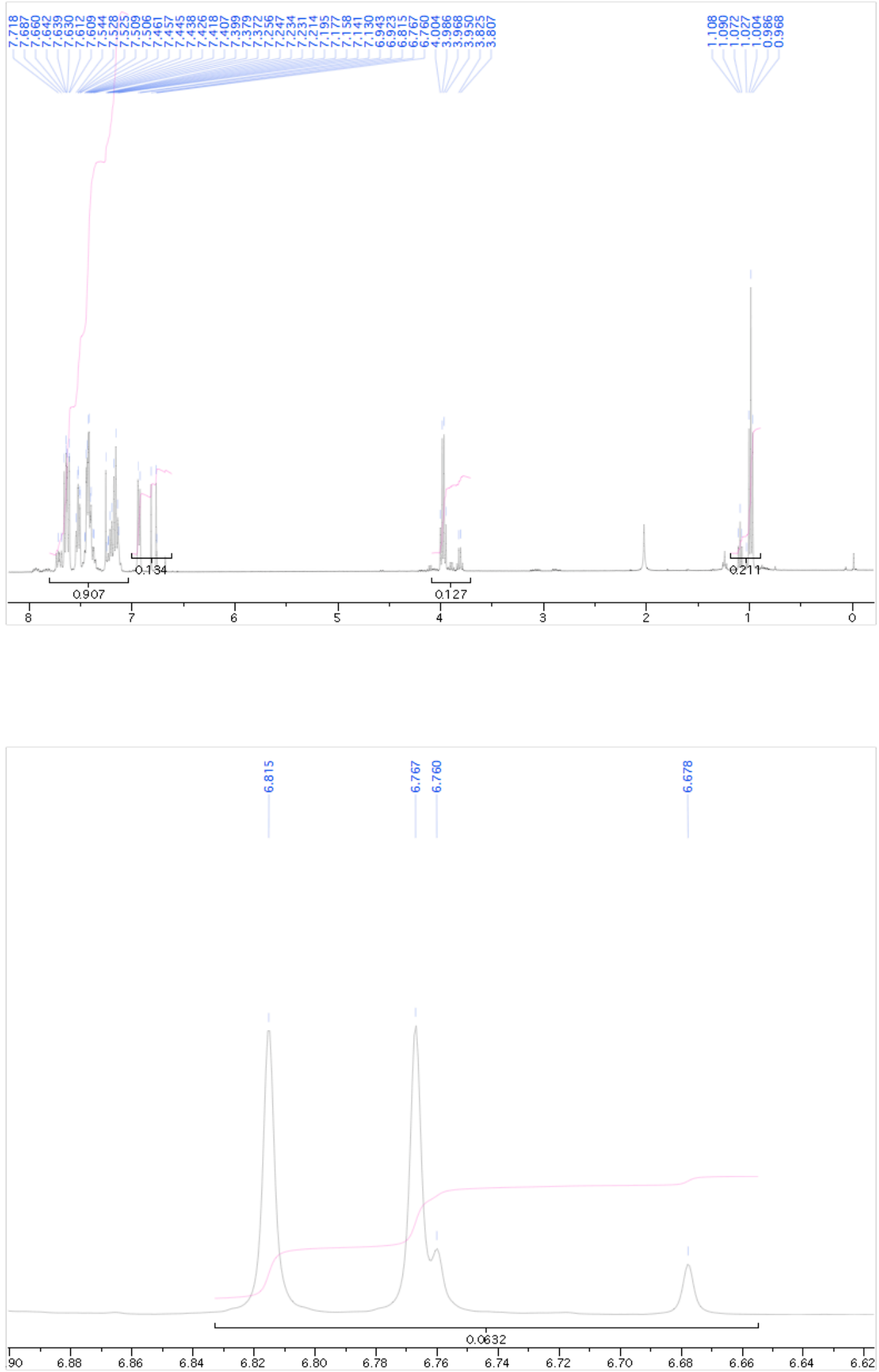
$3 \mathbf{i}\left({ }^{13} \mathrm{C}\right)$

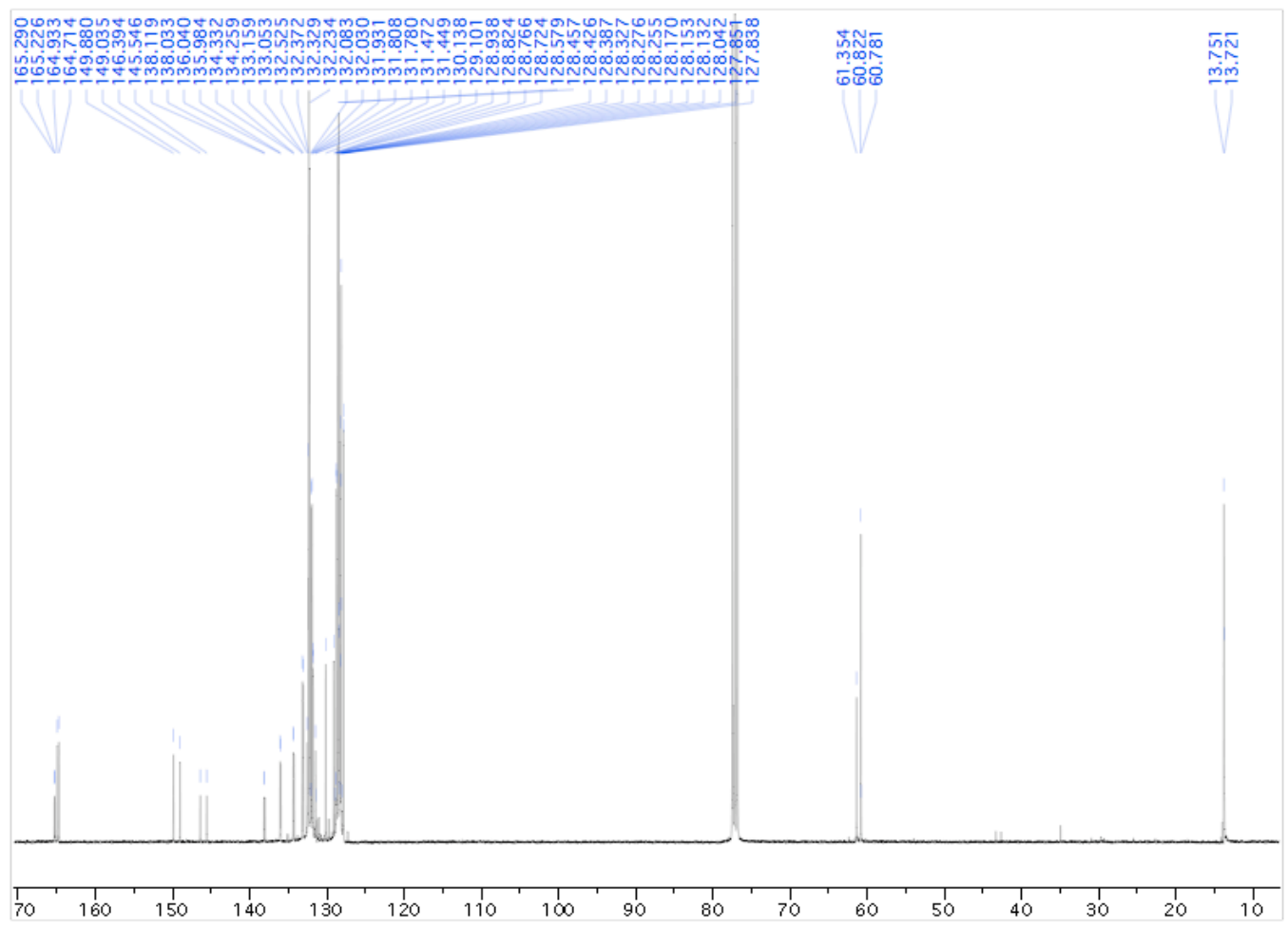

$3 \mathbf{i}\left({ }^{31} \mathrm{P}\right)$

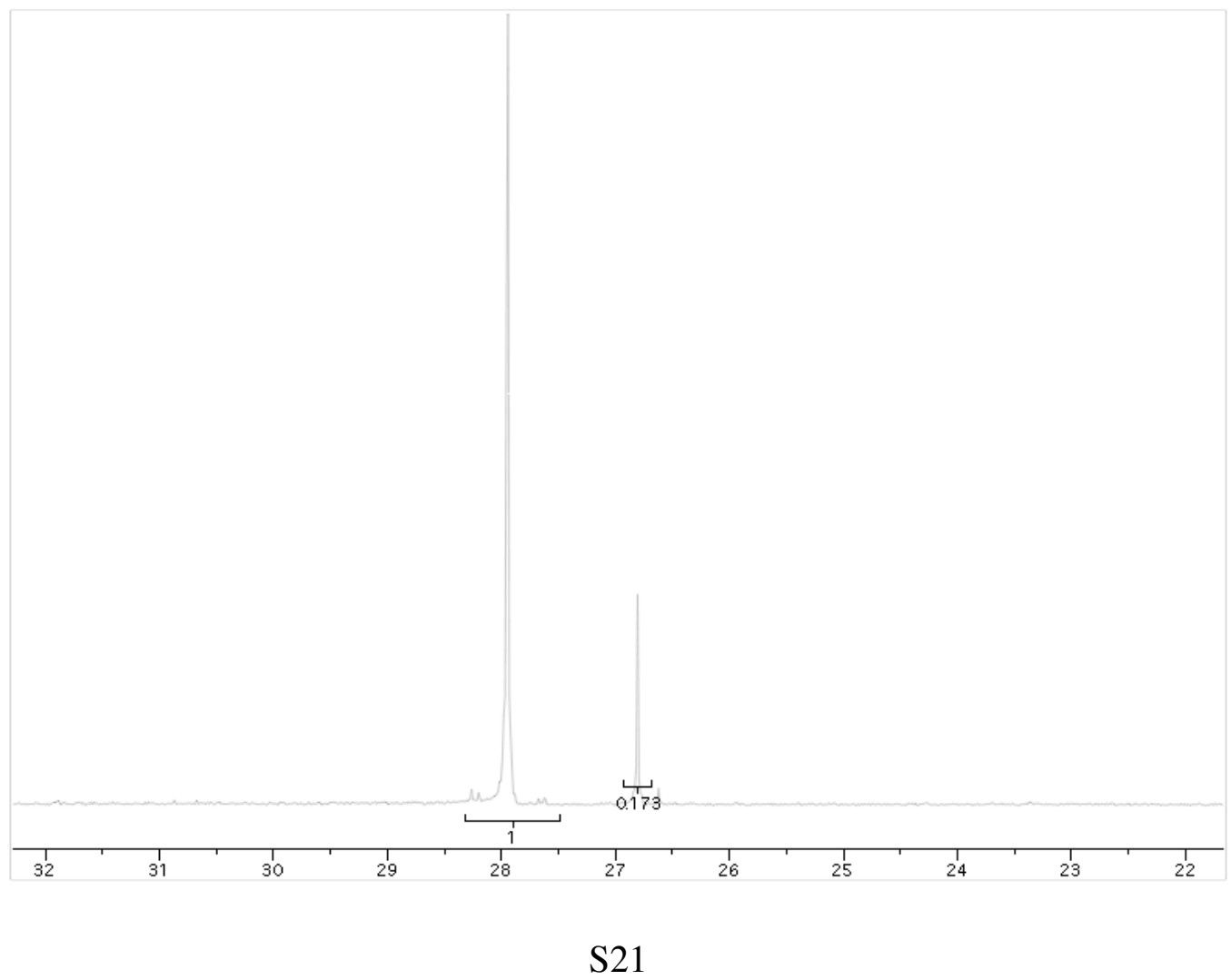


$5\left({ }^{1} \mathrm{H}\right)$

VIII

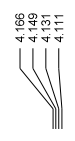

U|

|11
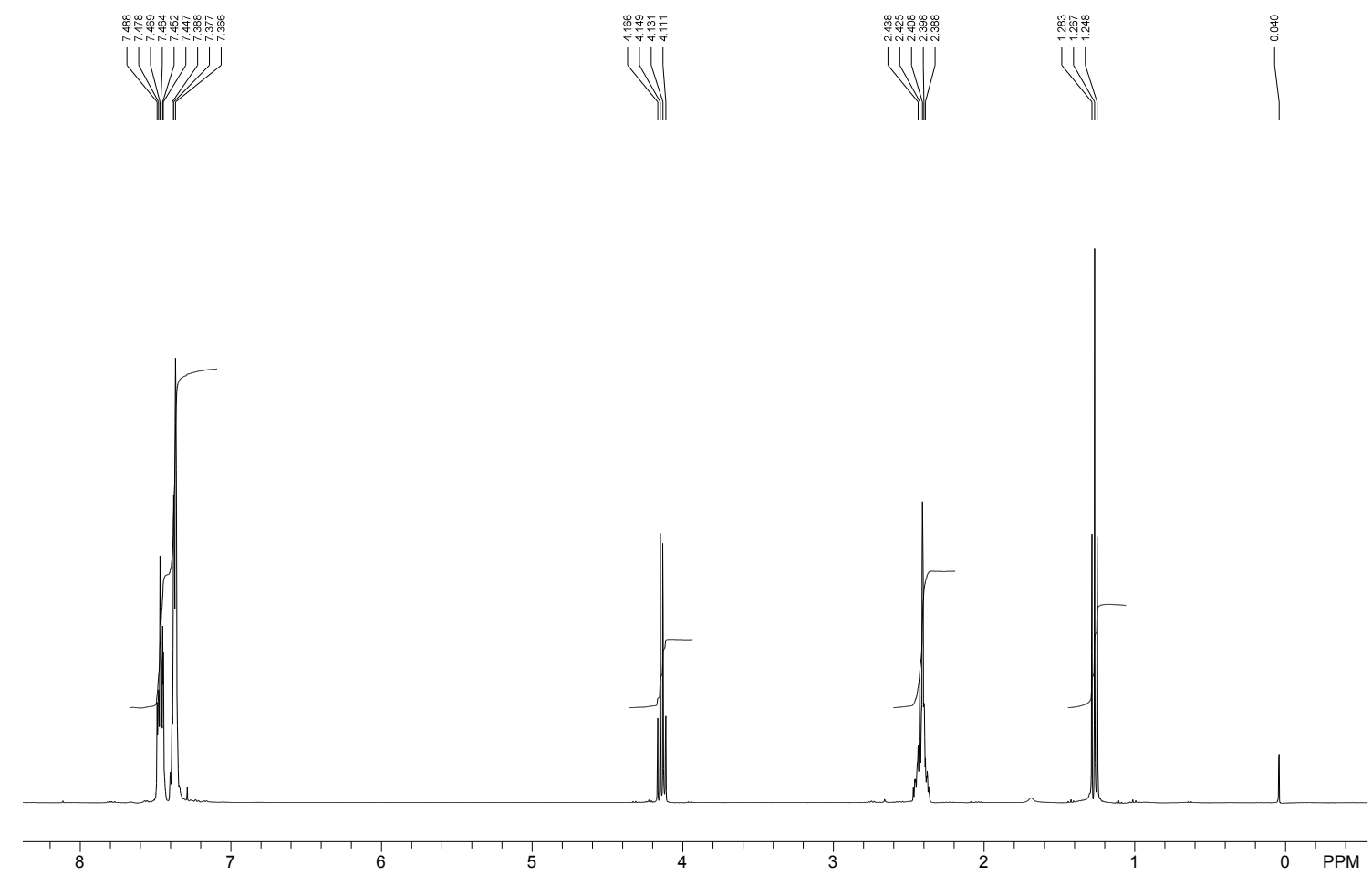

$5\left({ }^{13} \mathrm{C}\right)$
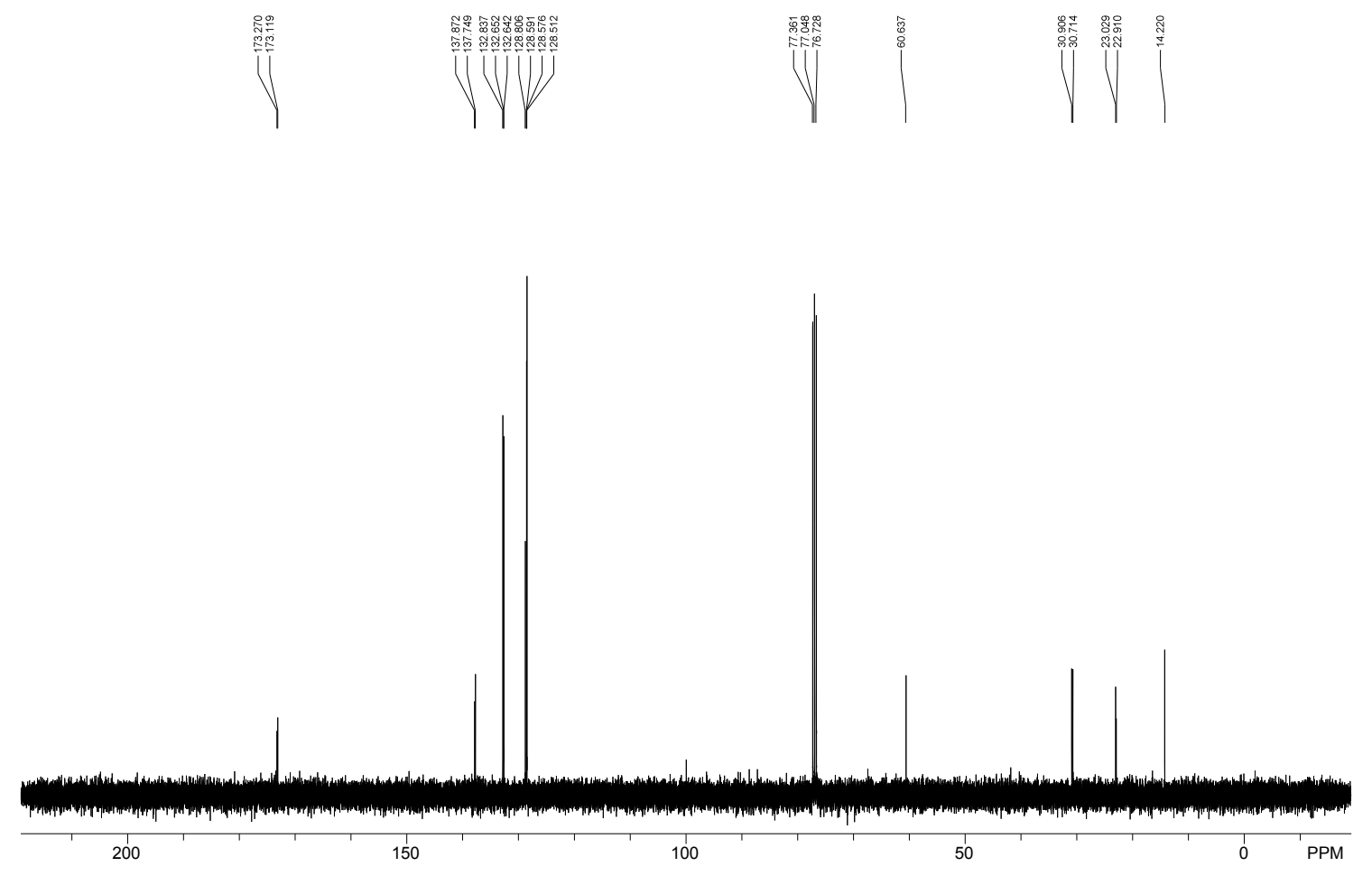
$5\left({ }^{31} \mathrm{P}\right)$

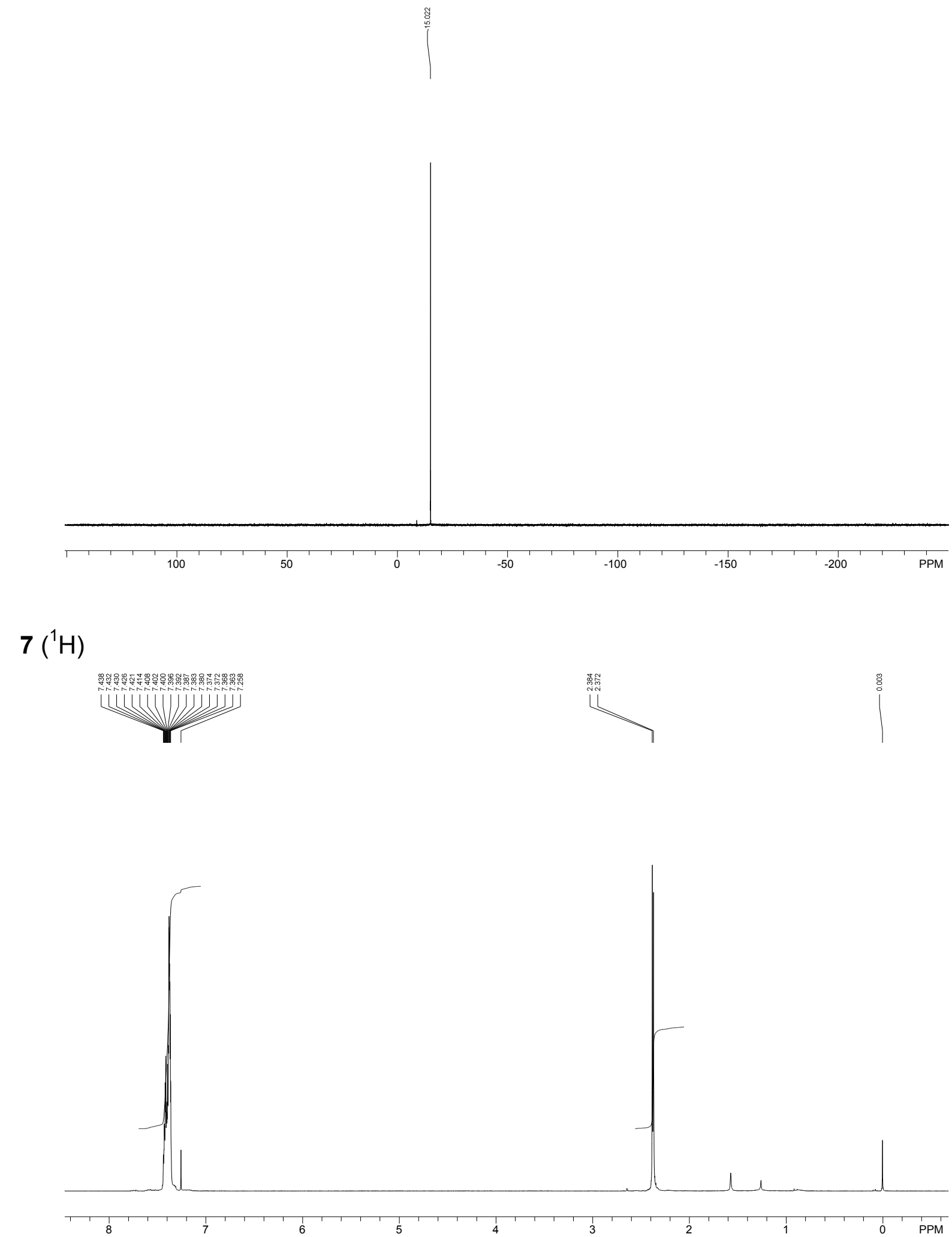


$7\left({ }^{13} \mathrm{C}\right)$
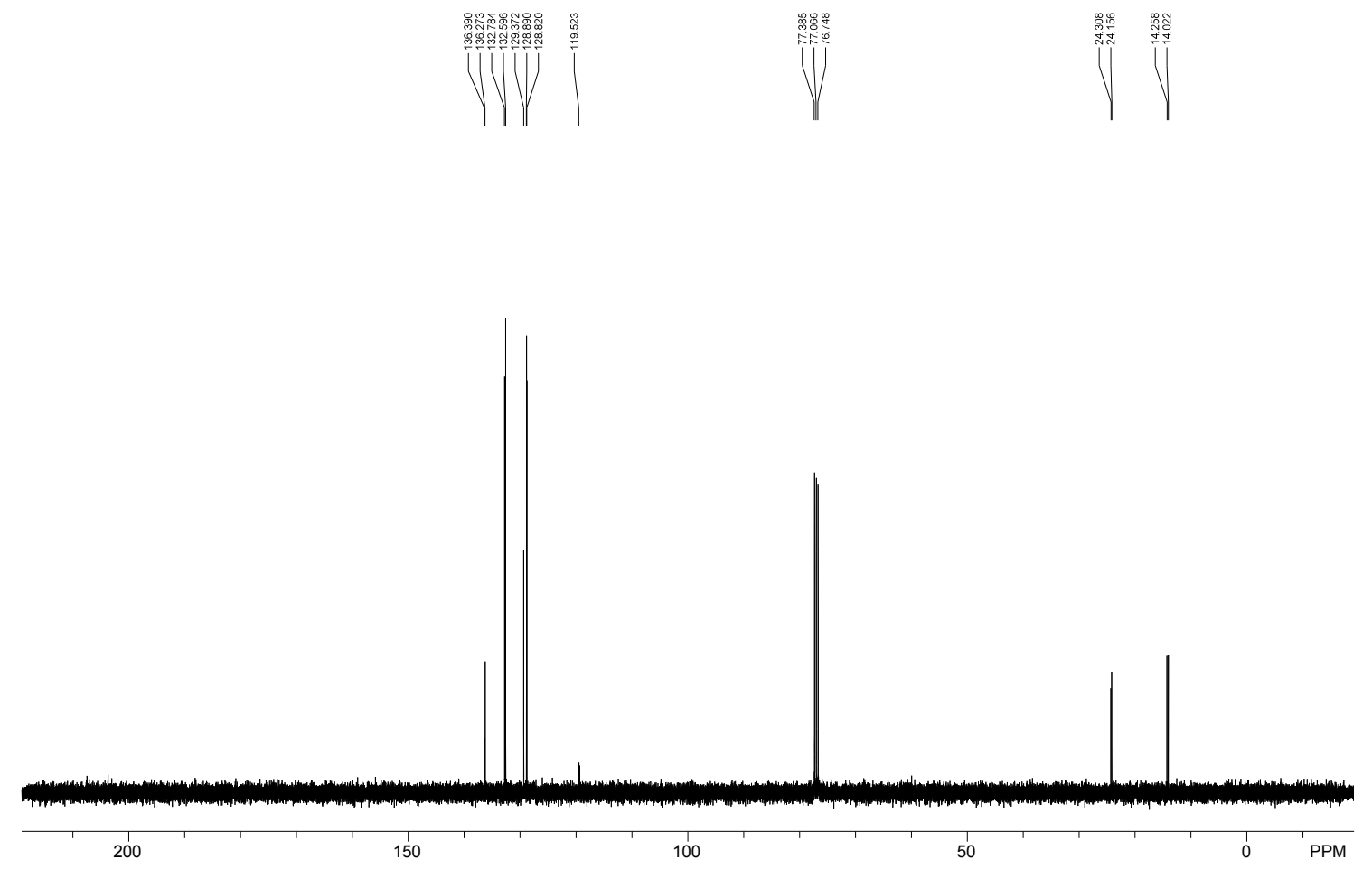

$7\left({ }^{31} \mathrm{P}\right)$

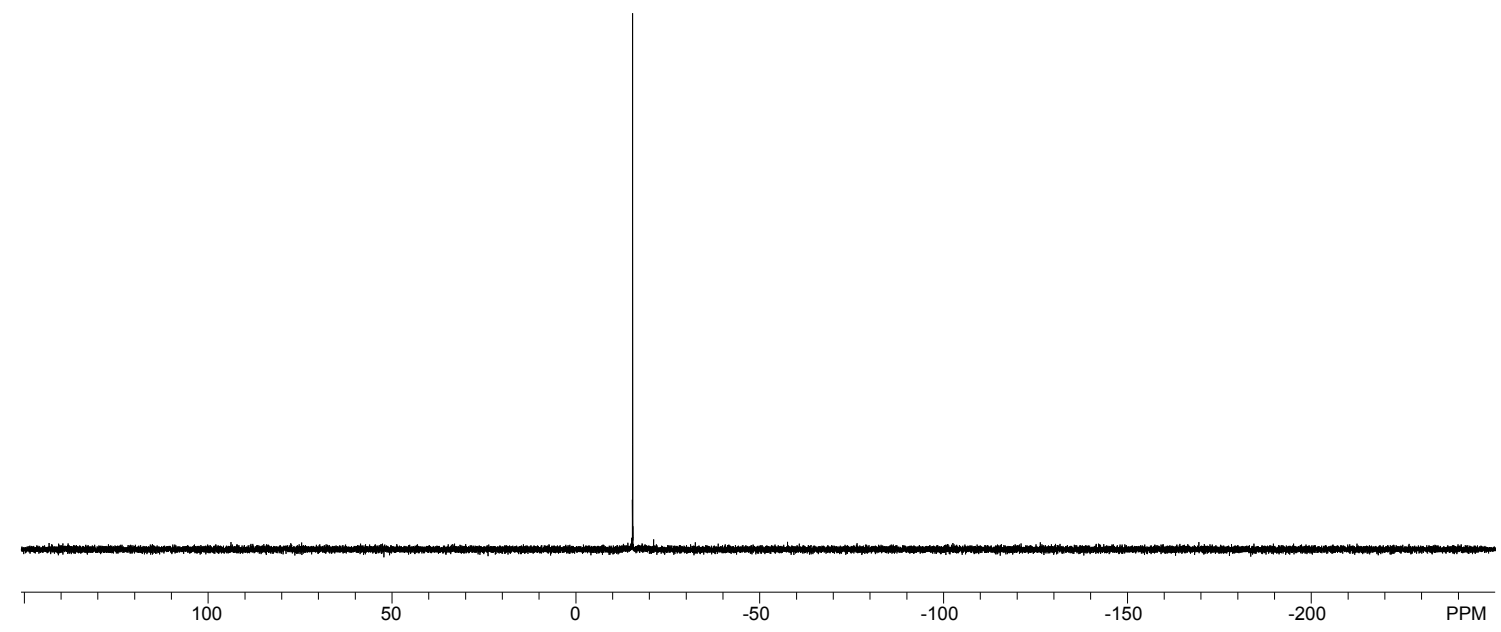

